\title{
ZINC-INDUCED FACILITATOR-LIKE family in plants: lineage-specific expansion in monocotyledons and conserved genomic and expression features among rice (Oryza sativa) paralogs
}

Felipe K Ricachenevsky ${ }^{1}$, Raul A Sperotto ${ }^{1}$, Paloma K Menguer ${ }^{2}$, Edilena R Sperb ${ }^{2}$, Karina L Lopes ${ }^{2}$, Janette P Fett ${ }^{1,2^{*}}$

\begin{abstract}
Background: Duplications are very common in the evolution of plant genomes, explaining the high number of members in plant gene families. New genes born after duplication can undergo pseudogenization, neofunctionalization or subfunctionalization. Rice is a model for functional genomics research, an important crop for human nutrition and a target for biofortification. Increased zinc and iron content in the rice grain could be achieved by manipulation of metal transporters. Here, we describe the ZINC-INDUCED FACILITATOR-LIKE (ZIFL) gene family in plants, and characterize the genomic structure and expression of rice paralogs, which are highly affected by segmental duplication.
\end{abstract}

Results: Sequences of sixty-eight ZIFL genes, from nine plant species, were comparatively analyzed. Although related to MSF_1 proteins, ZIFL protein sequences consistently grouped separately. Specific ZIFL sequence signatures were identified. Monocots harbor a larger number of ZIFL genes in their genomes than dicots, probably a result of a lineage-specific expansion. The rice ZIFL paralogs were named OsZIFL1 to OsZIFL 13 and characterized. The genomic organization of the rice ZIFL genes seems to be highly influenced by segmental and tandem duplications and concerted evolution, as rice genome contains five highly similar ZIFL gene pairs. Most rice ZIFL promoters are enriched for the core sequence of the Fe-deficiency-related box IDE1. Gene expression analyses of different plant organs, growth stages and treatments, both from our qPCR data and from microarray databases, revealed that the duplicated ZIFL gene pairs are mostly co-expressed. Transcripts of OsZIFL4, OsZIFL5, OsZIFL7, and OsZIFL12 accumulate in response to Zn-excess and Fe-deficiency in roots, two stresses with partially overlapping responses.

Conclusions: We suggest that ZIFL genes have different evolutionary histories in monocot and dicot lineages. In rice, concerted evolution affected ZIFL duplicated genes, possibly maintaining similar expression patterns between pairs. The enrichment for IDE1 boxes in rice ZIFL gene promoters suggests a role in Zn-excess and Fe-deficiency up-regulation of ZIFL transcripts. Moreover, this is the first description of the ZIFL gene family in plants and the basis for functional studies on this family, which may play important roles in $\mathrm{Zn}$ and Fe homeostasis in plants.

\section{Background}

Duplications are recurrent in the evolutionary history of plant genomes. Whole genome duplications (or polyploidy) are described for dicotyledons and monocotyledons [1-4]. It is estimated that the incidence of

\footnotetext{
* Correspondence: jpfett@cbiot.ufrgs.br

'Centro de Biotecnologia, Universidade Federal do Rio Grande do Sul, Av. Bento Gonçalves 9500, P.O.Box 15005, Porto Alegre, 91501-970, Brazil Full list of author information is available at the end of the article
}

polyploidy in angiosperms is $30-80 \%$, and ploidy changes may represent about $24 \%$ of speciation events [5]. Duplication generates two copies of each gene, and the fate of duplicated genes was first described by Ohno: one copy should maintain the ancient function and another copy should lose function (pseudogenization) or gain a new function (neofunctionalization) [6]. This model was improved, giving rise to the duplication-degenerationcomplementation (DDC) model, where the duplicated

\section{Biomed Central}


copies can have complementary functions that resemble the ancestral gene's function (subfunctionalization) [7]. The DDC model's predictions are believed to be more accurate than the previous model, since loss-of-function changes in regulatory regions are more likely to occur than gain-of-function mutations [7]. Other improvements of the basic model for duplicated gene retention, involving buffering of crucial functions via conversion and crossing-over, were recently proposed $[8,9]$.

Due to repetitive genome duplications, plants are likely to harbor relatively larger gene families, as compared to animal genomes [10]. It is well established that one whole-genome duplication occurred in the cereal lineage, estimated 70 million years ago (MYA), preceding the radiation of the major cereal clades by 20 million years or more $[3,11]$. Recently, comparing the genomic sequences of rice (Oryza sativa) and Sorghum bicolor, it was demonstrated that an early duplication occurred in the monocot lineage [4]. The duplication blocks cover at least $20 \%$ of the cereals transcriptome [4]. It was also shown that expression divergence between duplicate genes is significantly correlated with their sequence divergence [12]. After duplication, gene pairs rapidly diverge, and only a small fraction of ancient gene pairs do not show expression divergence [12]. However, for some genomic segments, concerted evolution homogenizes homologous sequences through unequal crossing-over and gene conversion, changing the estimated duplication age and gene divergence [9,13-15].

Rice was first described as having 18 duplicated segments which cover $65.7 \%$ of its genomic sequence, and several individual gene duplications [16]. More recent estimates account for 29 duplications in the rice genome, including 19 minor blocks that overlap with 10 major blocks [17]. A duplication block between chromosomes 11 and 12 has been extensively characterized in rice and other cereals, although the age of its birth is still controversial $[9,14,15,18,19]$. Rice is a model for cereal genomic and genetics studies, due to the availability of the genome sequences from two varieties, extensive gene annotation, and mutant resources [20-24]. Rice is also a major staple food, feeding nearly half of the world's population. However, it is a poor source of minerals such as iron $(\mathrm{Fe})$ and zinc $(\mathrm{Zn})$, the two mineral elements most commonly lacking in human diets $[25,26]$. Metal homeostasis in plants has been extensively studied in recent years, with a special focus on the transition metals $\mathrm{Zn}$ and Fe [27-29]. Thus, rice emerges both as a model species for physiological and molecular studies and as a candidate for biotechnological improvement aiming at $\mathrm{Zn}$ and $\mathrm{Fe}$ biofortification [30-32].

Both $\mathrm{Zn}$ and $\mathrm{Fe}$ are essential to mineral nutrition of plants. Zn has a key role in gene expression, cell development and replication, while Fe is necessary for photosynthesis, electron transport and other redox reactions [33]. Although essential, both can be toxic when in excess [34-37]. Several transporters involved in uptake and translocation inside the plant were described for Fe and $\mathrm{Zn}$ [35,38-43].

The ZINC-INDUCED FACILITATOR 1 gene (AtZIF1), described by Haydon and Cobbett, belongs to a new family of transporters, with three members in Arabidopsis thaliana: AtZIF1 (AT5G13740), AtZIFL1 (AT5G13750) and AtZIFL2 (AT3G43790) [34]. The AtZIF1 transporter is clearly involved in $\mathrm{Zn}$ homeostasis, as the loss-of-function atzif1 mutant has altered $\mathrm{Zn}$ distribution and its transcription is up-regulated by $\mathrm{Zn}$-excess [34]. Importantly, AtZIF1 proteins are expressed in the tonoplast, and probably are involved in transport of $\mathrm{Zn}, \mathrm{Zn}$ and a ligand or a ligand alone, to the vacuole [34]. Besides AtZIF1, only one similar protein had been previously characterized: the maize (Zea mays) $\mathrm{Zm}-\mathrm{mfs} 1$, which is induced by infection by the pathogens Cochliobolus heterostrophus and C. carbonum and to ultraviolet light [44]. This gene is highly expressed in the Les 9 disease lesion mimic background and in plant tissues engineered to express flavonoids or the avirulence gene avrRxv [44]. Both AtZIF1 and Zmmfs1 are part of the Major Facilitator Superfamily (MFS), which comprises the largest superfamily of secondary transport carriers found in living organisms and is subdivided in at least 29 families [45]. More recently, AtZIF1 and AtZIFL1 were described as quantitative trait loci (QTL) candidates for Zn concentrations in Arabidopsis seeds [46]. In barley (Hordeum vulgare), microarray analyses revealed that a ZIF1-like gene is expressed in the aleurone layer of seeds and its transcription increases in the embryo upon foliar $\mathrm{Zn}$ application [47]. Therefore, it is possible that ZIFL genes are involved in $\mathrm{Zn}$ translocation to the seeds.

In this work, we describe the ZIF-like (ZIFL) family of transporters. We identified 68 family members from plants and reconstructed their phylogenetic relationships. We also analyzed in detail the organization of ZIFL genes in the rice (Oryza sativa) genome: the motif composition, genomic organization, and promoter sequences. We analyzed the expression of OsZIFL genes in different plant organs and developmental stages, as well as in response to different stresses. This is the first attempt to describe the ZIFL gene family in plants, and the first expression analysis of these genes in rice.

\section{Results}

\section{ZIFL genes in plants}

We first used the AtZIF1, AtZIFL1 and AtZIFL2 sequences to query genomic databases to determine the distribution of this gene family among plant species. Two dicots, Vitis vinifera and Populus trichocarpa, one bryophyte, Physcomitrella patens, one lycophyte, Selaginella moellendorffii, 
and four monocots, Sorghum bicolor, Brachypodium distachyon, Oryza sativa and Zea mays had their genomes screened for ZIFL genes. All sequences found through this search plus the three Arabidopsis sequences were used to generate a Hidden Markov Model (HMM) profile to iteratively search the same genomes (see Methods). The final dataset consists of 66 genes coding for proteins already annotated (Additional File 1) and two unannotated proteins from Zea mays (Additional File 2).

All organisms queried contain ZIFL sequences, with predicted protein sequences ranging from 289 to 557 amino acids and an average of 468.4 amino acids per protein. All gene sequences begin with an initiation codon and end with a stop codon, except for the protein PpZIFL1, which lacks a small N-terminal portion (about 50 amino acids) and was included in the analyses. The overall structure contains 11 to 12 predicted transmembrane (TM) domains (Additional File 1 and Additional File 2), found in $63 \%$ of the proteins in our dataset. Fourteen putative proteins are predicted to have $10 \mathrm{TM}$ domains, and 11 proteins have seven to nine TM domains (Additional File 1 and Additional File 2).

Dicot species have a small number of ZIFL gene copies, with $V$. vinifera and $P$. trichocarpa showing five and four paralogs of ZIFL in their genomes, similar to the three members of the Arabidopsis ZIFL gene family [34]. Conversely, monocot species show a higher number of ZIFL genes, with $S$. bicolor having the highest number of members (14), followed by rice (13), B. distachyon (10) and Z. mays (10). P. patens and S. moellendorffii harbor two and seven ZIFL genes, respectively. Clearly, monocot species have a higher number of ZIFL family paralogs than dicots. The seven ZIFL genes found in $S$. moellendorffii seem to be closely related and not originated from the same expansion which originated the monocot ZIFL genes.

\section{ZIFL proteins are a distinct family of MFS transporters}

The ZIFL proteins are all part of the Major Facilitator Superfamily (MFS) clan of transporter proteins (Pfam number CL0015), composed by 22 families. They show similarity to the MFS_1 family (Pfam number PF07690), which is the largest family within the MFS clan. We used the MFS_1 HMM profile to isolate the MFS_1 proteins from all dicot and monocot genomes analyzed in this work. Phylogenetic trees reconstructing the evolutionary history of MFS_1 and ZIFL proteins for each species were generated using the neighbor-joining method (Additional File 3). We observed that in all cases the ZIFL proteins clustered in a separate group from all other MFS_1 members. This result could be an indication that ZIFL is a distinct family of MFS transporters.

Simmons et al suggested that sequences similar to Zm-mfs1 (ZmZIFL5 in Additional File 1 and throughout this work) could be a distinct group of MFS proteins found in plants [44]. This was based on comparison of signature sequences of nine plant sequences to bacterial and fungal MFS sequences. To confirm this hypothesis, we searched for signatures in the ZIFL HMM profile and aligned them to the MFS_1 HMM profile. We found the canonical MFS signature, located in the cytoplasmic loop between TM2 and TM3, as well as the antiporter signature in TM5 (Figure 1A). When aligning these signatures to the MFS_1 HMM profile, we noticed that the ZIFL MFS signature G-x(3)-D-[RK]-x-G-R-[RK] has a conserved tryptophan (W) before the first glycine position, which is not observed in MFS_1 (Figure 1A). The antiporter signature, $S-x(8)-G-x(3)-G-P-x(2)-G-G$, is also slightly different, having preference for serine in the first position, instead of glycine, as observed by Simmons et al (Figure 1A) [44]. The presence of these conserved positions indicates that ZIFL transporters share structural and functional similarities with MFS antiporters, although they show specific features that are not common to other MFS proteins.

The ZIFL sequences also show signatures that are not shared with MFS_1 proteins. The conserved positions in the loop between TM8 and TM9, [RK]-x(2)-G-P-[IV]-x (3)-R, previously reported by Simmons et al, were confirmed in our dataset with a few changes (Figure 2B) [44]. Importantly, we found two new conserved signatures that are specific for the ZIFL proteins. One of them is a cysteine (Cys)-containing motif C-[PS]-G-C in the cytoplasmic $\mathrm{N}$-terminal loop of ZIFL proteins, and the second one is a histidine (His)-containing motif [PQ]-E-[TS]-[LI]-H-x-[HKLRD] in the cytoplasmic loop between TM6 and TM7, before the beginning of a variable region (Figure 2B; see below). From our dataset of 68 ZIFL proteins, 58 have the Cys motif, with only three proteins showing a serine residue in the second position instead of a proline (Additional File 4). For the histidine motif, 61 ZIFL proteins have the conserved residues (Additional File 4). From these, 45 have the most conserved residues P-E-T-L-H-x-H, while the other 16 ZIFL members contain the same motif with no more than one residue substitution (Additional File 4). Considering that the MFS_1 family has 56,680 proteins with very low overall similarity between them, and that ZIFL proteins share both high similarity and unique signatures, we suggest that ZIFL proteins comprise a distinct family of transporters.

\section{ZIFL gene expansion is lineage specific}

To address the hypothesis of a lineage specific expansion of ZIFL genes in monocot species, we generated an alignment using the amino acid sequences of the 68 ZIFL genes found and reconstructed the phylogenetic relationships of these protein sequences using two 


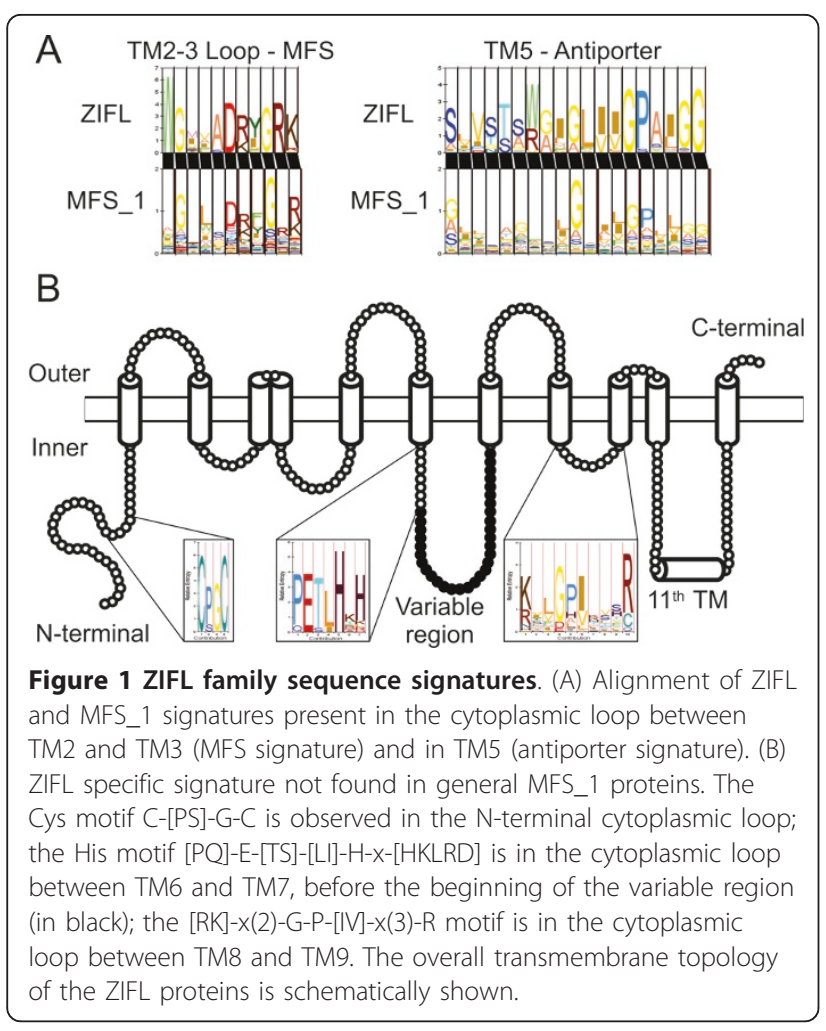

methods: neighbor-joining and bayesian analysis (Figure 2). Although some nodes are not in agreement comparing the two methods, our bootstrap values and posterior probabilities support all the major nodes of the tree, indicating that the reported group relationships are reliable (Figure 2).

Proteins from bryophyte and lycophyte species grouped together, with paralogs from each species in a separate cluster. The ZIFL proteins from dicots also formed a distinct group (Figure 2). However, there was no clear separation into sub-groups of orthologous sequences within the dicots group (Figure 2). Species-specific gene duplications are observed in Arabidopsis (AtZIF1 and AtZIFL1), $V$. vinifera (VvZIFL2 and VvZIFL3; VvZIFL4 and VvZIFL5) and P. trichocarpa (PtZIFL1 and PtZIFL4) (Figure 2).

The ZIFL paralogs from monocot species were grouped in three distinct groups, named Monocot I, Monocot II and Monocot III. All three ZIFL protein groups from the monocots contain paralogs from the four species included in our analysis. The Monocot I group contains 17 ZIFL proteins, while the Monocot II and Monocot III groups contain 15 proteins each (Figure 2). Both the number of sequences found in monocot species and the tree topology strongly suggest that the ZIFL gene family experienced an expansion in the monocot lineage, and that the last common ancestor of the monocots already had ZIFL paralogs of the three groups (Figure 2). Thus, the split of the four monocot species used in this work probably occurred after the expansion of the ZIFL family observed in monocots, and this expansion is not shared with other plant lineages.

\section{ZIFL paralogs are unequally distributed in the rice genome}

The identification of the ZIFL gene chromosome locations revealed that they are not evenly distributed in the rice genome, but rather arranged in clusters (Additional File 5). The same trend is observed in S. bicolor and B. distachyon, but not in Z. mays (Additional File 5). Rice ZIFL genes were named ZIFL1 to 13 based on their genomic locations. Two ZIFL genes, OsZIFL1 and OsZIFL2 are located in chromosome 1 , and OsZIFL3 is located in chromosome 7. OsZIFL4, OsZIFL5, OsZIFL6, OsZIFL7 and OsZIFL8 are found in chromosome 11, while OsZIFL9, OsZIFL10, OsZIFL11, OsZIFL12 and OsZIFL13 are located in chromosome 12. Interestingly, the ZIFL genes arranged in tandem in chromosomes 11 and 12 are closely related, with OsZIFL4 being very similar to OsZIFL9 (92\% of identity), OsZIFL5 to OsZIFL10 (95\%), OsZIFL6 to OsZIFL11 (82\%), OsZIFL7 to OsZIFL12 (85\%) and OsZIFL8 to OsZIFL13 (73\%) (Table 1). We used the GATA tool to align the $100 \mathrm{~kb}$ regions that include OsZIFL genes in chromosomes 11 and 12 (hereafter Os11 and Os12; Figure 3A). The regions of chromosomes 11 and 12 where these genes are located have already been described as a recent segmental duplication in the rice genome, what would explain the high number of matches between these regions (Figure 3A) [18,48]. However, the same duplication was recently found in S. bicolor, indicating that this segmental duplication is ancient to the split of these species $[14,15]$. We observed that $S$. bicolor chromosomes 5 and 8 (hereafter Sb05 and Sb08), which are homologous to rice chromosomes 11 and 12 (Os11 and Os12), harbor three and two ZIFL genes, respectively (Figure 3B) [14]. An incomplete sequence related to ZIFL is also found in chromosome 8 (Sb08g001390; Figure 3B). It is possible to observe that an inversion has occurred when comparing the orientation of ZIFL regions in $\mathrm{Sb} 05$ and Sb08 (Figure 3B). The alignment between rice and S. bicolor homologous chromosomes Os11 with Sb05 and Os12 with Sb08 demonstrate that the S. bicolor ZIFL region in $\mathrm{Sb08}$ is inverted, since the alignment of Os11 with Sb05 is in direct orientation (Figure 3C) while the alignment of Os12 with Sb08 is in reverse (Figure 3D). Therefore, although in homologous regions, the ZIFL gene cluster in Sb08 is differentially oriented in relation to rice.

\section{OsZIFL genes organization is highly conserved}

We aligned the genomic and coding sequence (CDS) of each ZIFL gene from rice and determined the exonintron organization (Figure 4). The exon sizes of each 


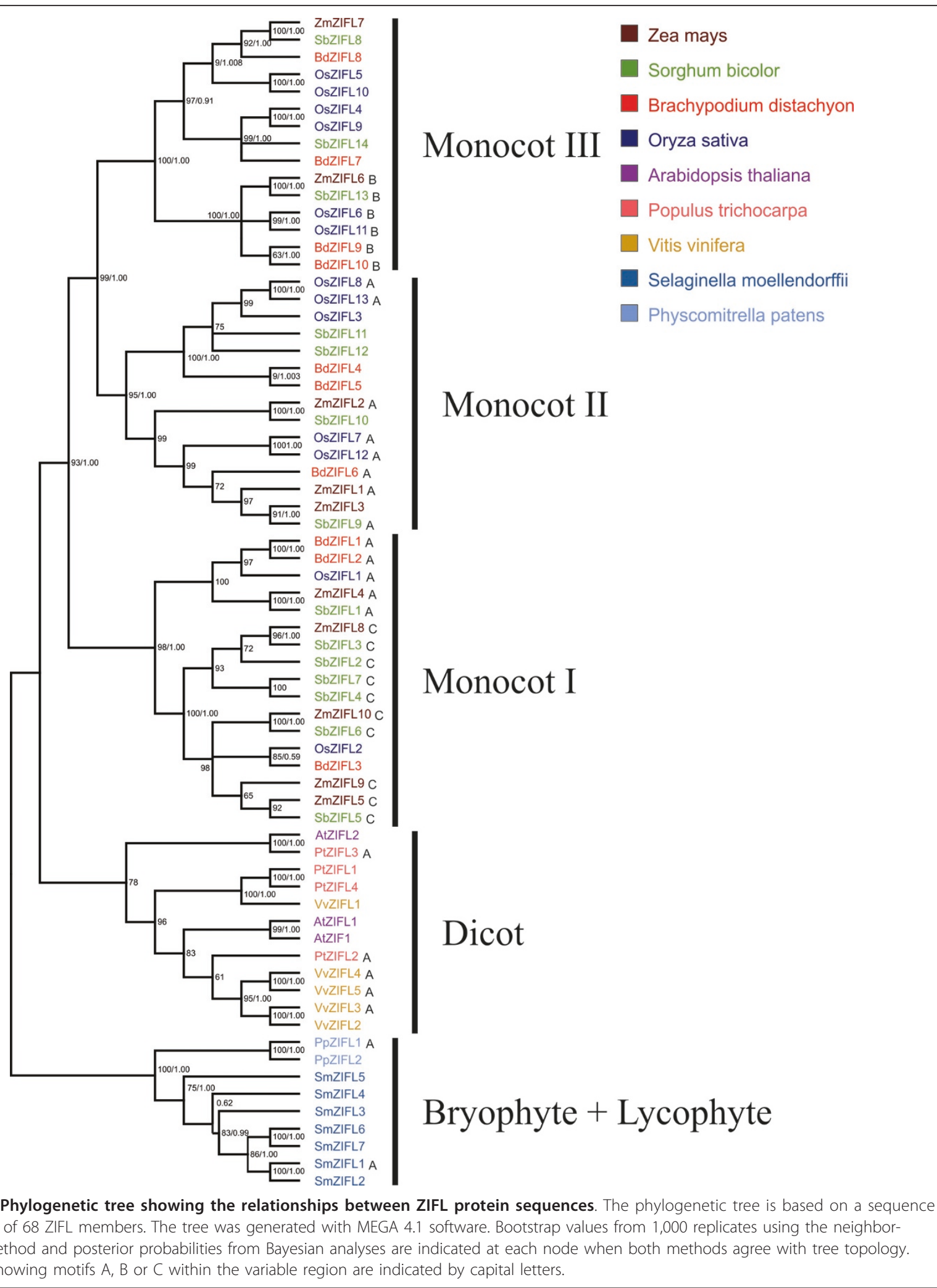


Table 1 Rice ZIFL sequence identity at the amino acid level

\begin{tabular}{|c|c|c|c|c|c|c|c|c|c|c|c|c|}
\hline & OsZIFL1 & OsZIFL2 & OsZIFL3 & OsZIFL4 & OsZIFL5 & OsZIFL6 & OsZIFL7 & OsZIFL8 & OsZIFL9 & OsZIFL10 & OsZIFL11 & OsZIFL12 \\
\hline OsZIFL2 & 57 & & & & & & & & & & & \\
\hline OsZIFL3 & 44 & 44 & & & & & & & & & & \\
\hline OsZIFL4 & 53 & 53 & 49 & & & & & & & & & \\
\hline OsZIFL5 & 55 & 55 & 50 & 74 & & & & & & & & \\
\hline OsZIFL6 & 51 & 52 & 46 & 66 & 69 & & & & & & & \\
\hline OsZIFL7 & 51 & 52 & 43 & 56 & 58 & 50 & & & & & & \\
\hline OsZIFL8 & 48 & 47 & 60 & 54 & 54 & 48 & 47 & & & & & \\
\hline OsZIFL9 & 54 & 55 & 44 & $92^{\mathrm{a}}$ & 72 & 60 & 53 & 49 & & & & \\
\hline OsZIFL10 & 55 & 52 & 48 & 72 & $95^{\mathrm{a}}$ & 68 & 57 & 53 & 70 & & & \\
\hline OsZIFL11 & 47 & 49 & 42 & 61 & 62 & $82^{\mathrm{a}}$ & 49 & 44 & 59 & 62 & & \\
\hline OsZIFL12 & 54 & 54 & 56 & 62 & 66 & 58 & $86^{a}$ & 61 & 62 & 65 & 54 & \\
\hline OsZIFL13 & 40 & 37 & 40 & 39 & 40 & 35 & 48 & $73^{a}$ & 39 & 40 & 39 & 47 \\
\hline
\end{tabular}

${ }^{a}$ Rice ZIFL gene pairs from chromosomes 11 and 12 .

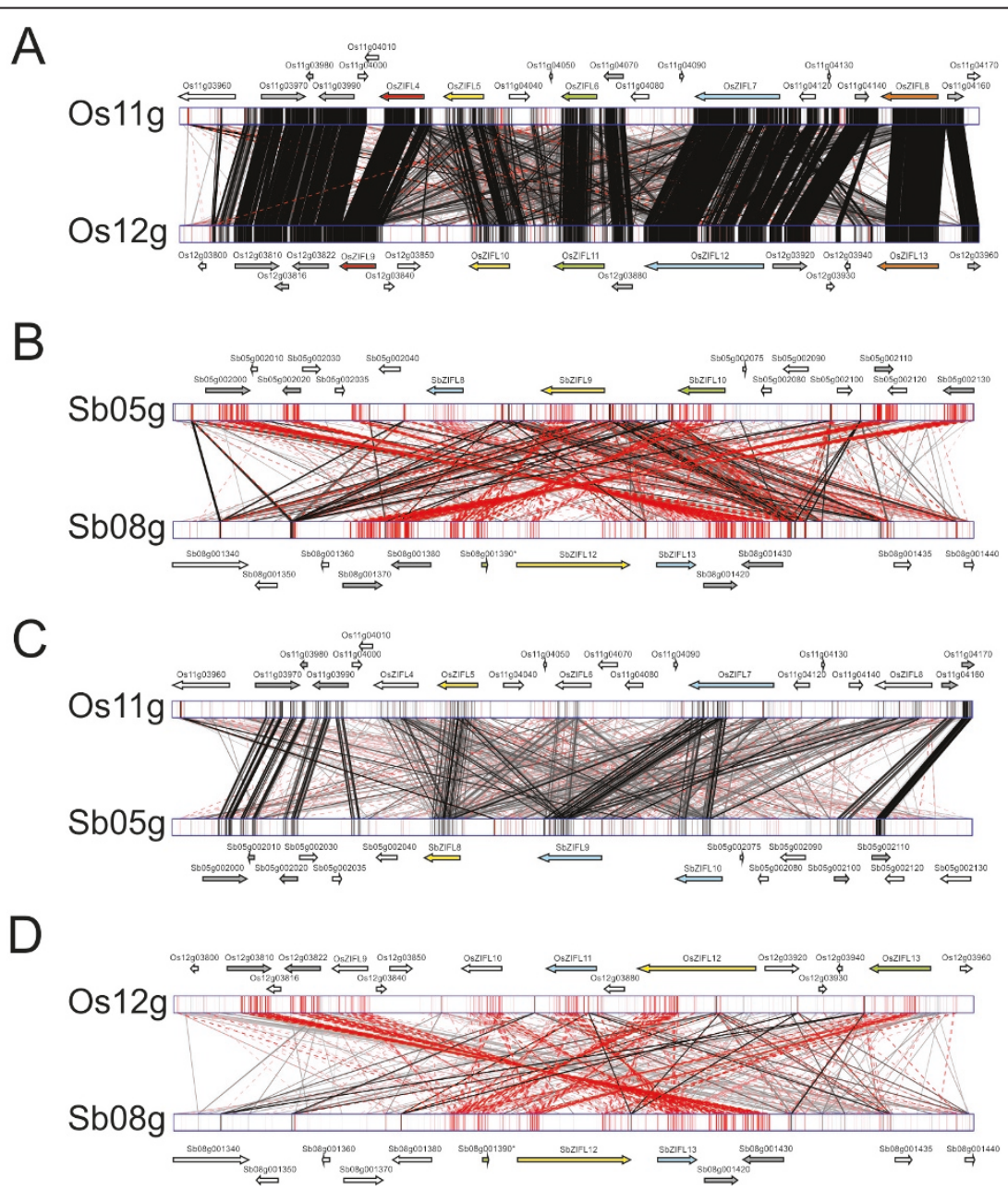

Figure 3 Genomic alignment obtained with the GATA tool using $100 \mathrm{~kb}$ regions containing rice or S. bicolor ZIFL genes. Black lines indicate a direct match, while red lines indicate an inverted match. Gene positions and orientations are denoted by arrows. ZIFL genes marked with the same color in both species indicate the closest homologs. Duplicated non-ZIFL genes are shown in gray. (A) Rice chromosomes 11 and 12. (B) S. bicolor chromosomes 5 and 8. (C) Rice chromosome 11 and S. bicolor chromosome 5. (D) Rice chromosome 12 and S. bicolor chromosome 8 . 


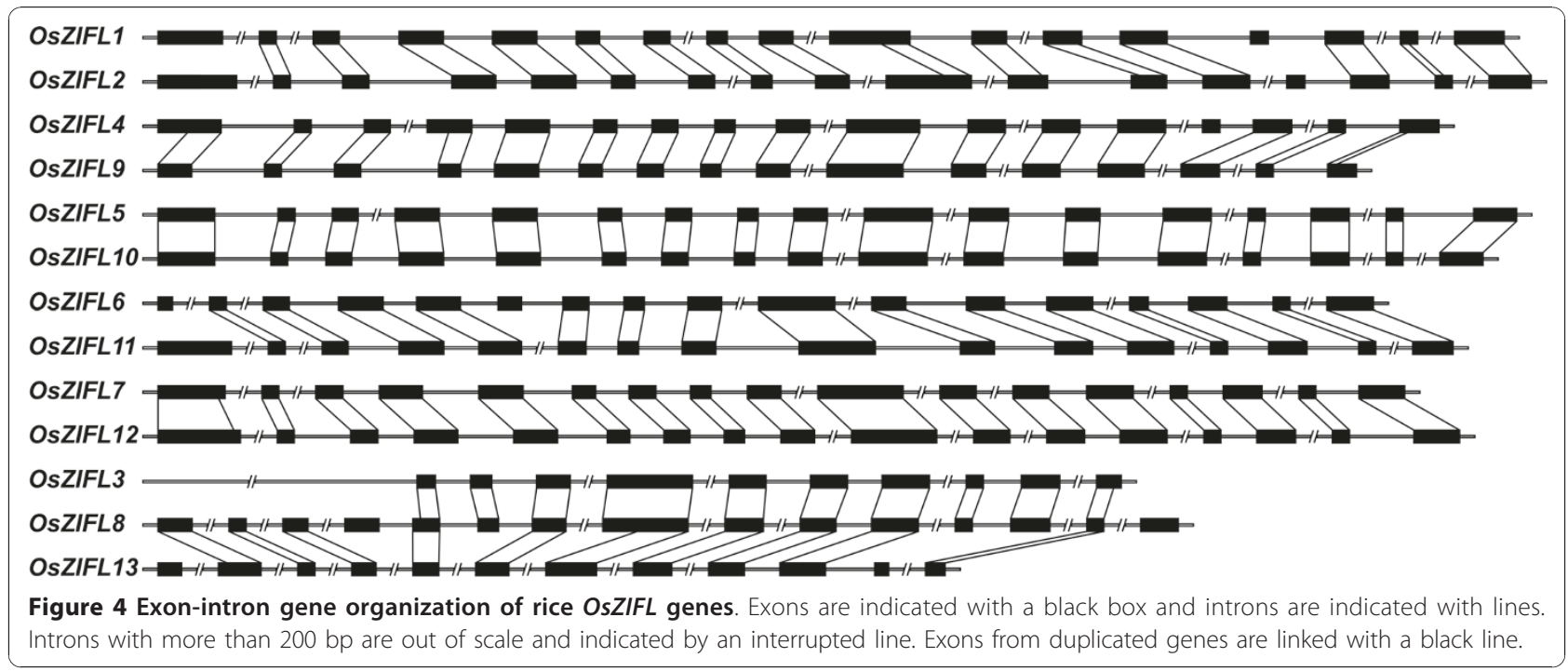

gene pair, OsZIFL4-OsZIFL9, OsZIFL5-OsZIFL10, OsZIFL6-OsZIFL11, OsZIFL7-OsZIFL12 and OsZIFL8OsZIFL13 are nearly identical, with very few variations in sequences. We observed that OsZIFL1 and OsZIFL2 are probably originated from duplication, since they share a similar exon-intron organization. However, their amino acid sequences are only $57 \%$ identical (Table 1 ). This duplication probably occurred in the common ancestor of monocots, as orthologs from S. bicolor, B. distachyon and Z. mays were found for both OsZIFL1 and OsZIFL2 (Figure 2). OsZIFL3 is suggested to be originated from a partial duplication of the OsZIFL8-OsZIFL13 pair last common ancestor (Figures 2 and 4), and shares more identities to OsZIFL8 sequence $(60 \%)$ than to OsZIFL13 (40\%). Thus, it is clear that duplications were of major importance in the ZIFL family expansion in rice, especially the segmental duplication observed in chromosomes 11 and 12 .

Protein motif composition reveals a variable region in the ZIFL family

We aligned the 13 rice ZIFL proteins and observed that they share large similarity (Additional File 6 and Table 1). To search for functional sites shared by OsZIFL putative proteins, we used MEME (http:// meme.nbcr.net/) to identify conserved motifs in their amino acid sequences [49]. We found eleven motifs shared by almost all 13 OsZIFL proteins, with few exceptions (Table 2, Figure 5A). Seven motifs matched the general MFS_1 motif in InterProScan (http://www. ebi.ac.uk/Tools/InterProScan/) (motifs 1, 2, 4, 5, 6, 7 and 9), while four showed no hits (motifs $3,8,10$, and

Table 2 Conserved motifs found in ZIFL protein sequences

\begin{tabular}{|c|c|c|c|}
\hline Motif & Width & Assign & Amino acid sequence \\
\hline 1 & 50 & $\mathrm{MFS}^{\mathrm{a}}$ & NWPLMSSIILYCVFSFHDMAYSEIFSLWAESDRKYGGLSFSSEDVGQVLA \\
\hline 2 & 50 & $\mathrm{MFS}^{\mathrm{a}}$ & QPAEKYPNVFSEKSIFGRFPYFLPCLCISVFAAWLISCIWLPETLHKHK \\
\hline 3 & 41 & No hit & LPISSLFPFLYFMIRDLHVAKREEDIGFYAGFVGASYMIGR \\
\hline 4 & 50 & $\mathrm{MFS}^{\mathrm{a}}$ & LQNNAVPQDQRGTANGIATTAMSFFKAIAPAGAGVLFSWAQKRQHAAFFP \\
\hline 5 & 50 & $M S^{a}$ & GASLLVYQLFIYPWVHKVLGPINSSRIAAILSIPILCTYPFMTHLSGPWL \\
\hline 6 & 50 & $\mathrm{MFS}^{\mathrm{a}}$ & RFLLGALNGMLGPIKAYSIEVCRPEHQALGLSIVSTAWGIGLWGPAIGG \\
\hline 7 & 29 & $\mathrm{MFS}^{\mathrm{a}}$ & PVIVFSIFSWIFNTLFGLSTKYWMAITT \\
\hline 8 & 21 & No hit & HDGCPGCAMERRKEEHKGIPY \\
\hline 9 & 15 & $\mathrm{MFS}^{\mathrm{a}}$ & ASIFWGIVADRIGRK \\
\hline 10 & 28 & No hit & GDQMVFFMLNVTEVIGLMLTFKPFLAVP \\
\hline 11 & 21 & No hit & VLNIASMMKNNLAVTIITGTN \\
\hline$A^{b}$ & 21 & No hit & NSVEALEEHLMDPNEEENENE \\
\hline$B^{b}$ & 15 & No hit & IKRIKELPSQQAYWD \\
\hline$C^{b}$ & 11 & No hit & EELEAQVGGSN \\
\hline
\end{tabular}

${ }^{a}$ MFS (Major Facilitator Superfamily Antiporter)

${ }^{\mathrm{b}}$ Analysis performed with whole set of ZIF proteins (68 sequences) 


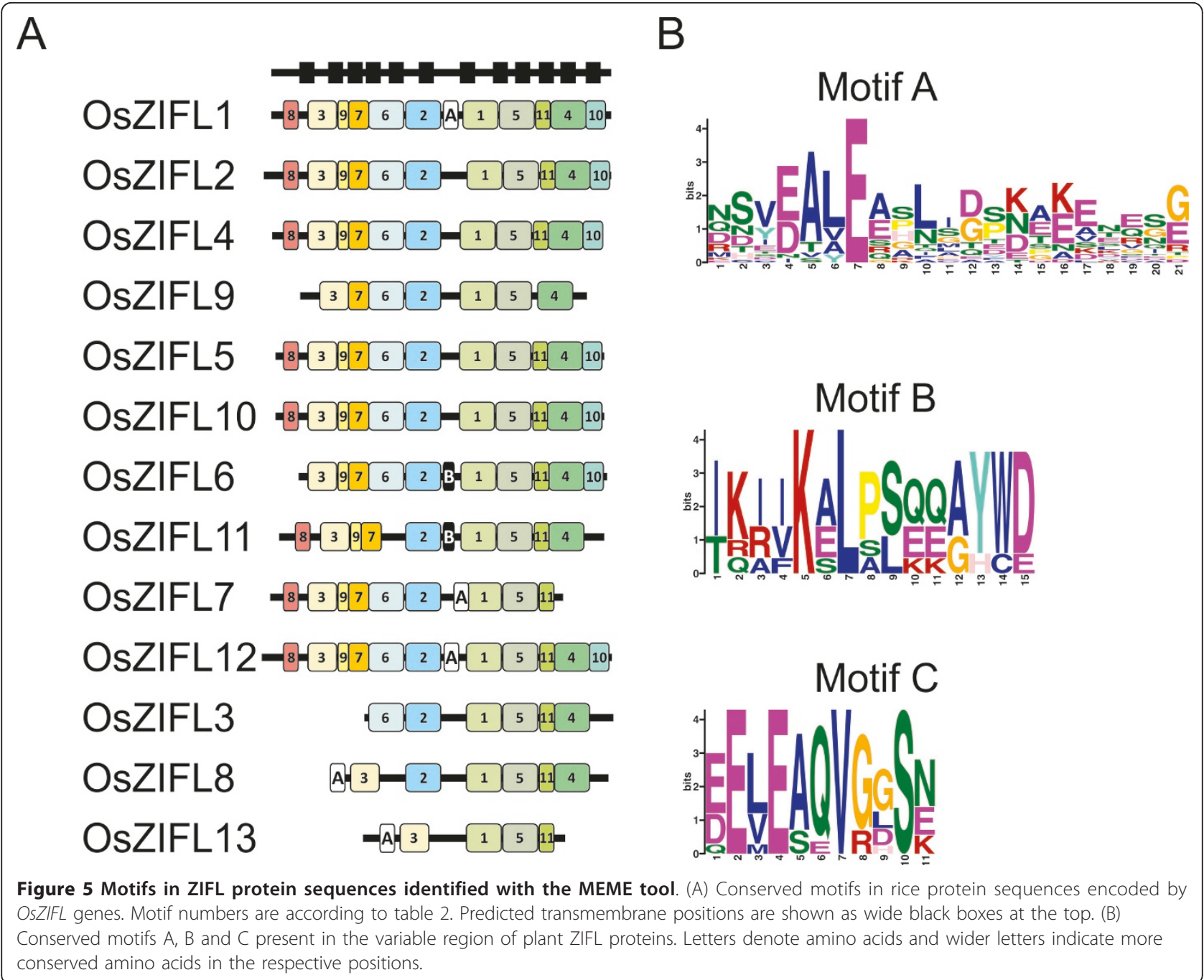

11) (Table 2). The ZIFL signatures Cys motif and His motif are located within the motif 8 and motif 2 , respectively (Table 2).

OsZIFL1, OsZIFL2, OsZIFL4, OsZIFL5, OsZIFL10 and OsZIFL12 have all eleven motifs, while the duplicated pair OsZIFL8-OsZIFL13 and their duplicated copy in chromosome 7 (OsZIFL3) lack several motifs (Figure 5A). Some of these motifs are located in regions predicted to be transmembrane (Figure 5A, black boxes at the top). Further characterization is needed to determine if the duplicated rice ZIFL genes are becoming pseudogenes or acquiring new functions.

The OsZIFL4 duplicated copy OsZIFL9 lacks the $\mathrm{N}$-terminal motif 8 and the C-terminal motif 10; OsZIFL6 lacks motif 8 and its duplicated copy OsZIFL11 lacks motif 6 and motif 10; the duplicated pair OsZIFL7 and OsZIFL12 only differ by the C-terminal motifs 4 and 10 , which are absent in OsZIFL7 (Figure 5A). These differences suggest a divergence process between duplicated pairs. Moreover, it is clear that the central motifs are more conserved than those located at the $\mathrm{N}$ - and $\mathrm{C}$-terminal regions of OsZIFL proteins (Figure 5A).

We also observed a variable region between motifs 1 and 2 which did not show significant pattern conservation in OsZIFL proteins (Figure 5A). This region is located between transmembrane regions 6 and 7 (considering 12 TM proteins) and is a cytoplasmic loop according to Conpred II predictions (Figure 1B). The variable region is preceded by the conserved His motif P-E-T-L$\mathrm{H}-\mathrm{x}-\mathrm{H}$ (Figure 1B). Variable regions are found in transporters and could be involved in transport or sensing functions [50,51]. The whole set of 68 ZIFL proteins used in this work was submitted to MEME analysis to find any conserved motifs specifically in the variable region. Three motifs were found in this region and named motifs A, B and C (Table 2; Figure 5B). None matched any known motif in the InterPro database (Table 2). We indicated proteins that contain each motif 
in our phylogenetic tree (Figure 2) and showed their positions in rice ZIFL protein sequences (Figure 5A). Rice ZIFL proteins contain motifs $\mathrm{A}$ and $\mathrm{B}$ in their variable region, but not motifs $\mathrm{C}$.

Motif A is present in proteins from the Monocot I, Monocot II, Dicot and Bryophyte-Lycophyte groups (Figure 1). This motif shows low amino acid conservation (Figure 5B). The negatively charged glutamic acid (E) residue in the seventh position of the motif is the most conserved residue. Conserved negatively charged residues are also found in the fourth position (glutamic or aspartic acid, E or D). Between these positions, two non-polar residues, alanine (A) and leucine (L) are also conserved (Figure 5B). Other positions containing a positively-charged residue of lysine (K), a negatively charged glutamic acid (E), and residues of leucine (L) and glycine (G), although less conserved, are present (Figure 5B). Charged positions could be involved in transporter specificity, as already described for cation diffusion facilitator (CDF) proteins [52]. Motif B is shared only by a sub-group of six proteins from monocot II (Figure 2). The fifth and seventh positions of this motif contain one positively charged residue and one hydrophobic residue, lysine $(\mathrm{K})$ and leucine $(\mathrm{L})$ (Figure 4B). Polar residues of serine (S), glutamine (Q) and tyrosine $(\mathrm{Y})$, non-polar tryptophan $(\mathrm{W})$ and proline (P) are also observed (Figure 4B). The motif C is common to 10 proteins from the Monocot I group (Figure 2), and is similar to motif A, showing the two glutamic acids (E) separated by one instead of two nonpolar residues (Figure 5B). However, since only a small number of proteins share motifs $B$ and $C$, we should be cautious on making assumptions about the functionality of conserved amino acids found in these motifs, as their conservation could be an effect of phylogenetic relatedness and not of evolutionary constraints.

Importantly, it is possible to observe the high divergence of the variable region even when comparing these three motifs. The variability is much higher in this region than in the whole sequence of ZIFL proteins, as MEME analysis revealed several motifs shared by all the 68 ZIFL proteins (data not shown). Therefore, these motifs in the cytoplasmic loop could be involved in specific functions of different ZIFL proteins.

\section{Expression of OsZIFL genes in rice vegetative and reproductive organs}

We analyzed the expression levels of OsZIFL transcripts in several rice organs by qPCR, including roots, culms and shoots (vegetative tissues); flag-leaves and whole panicles (reproductive tissues), both during R3 (panicle exertion), R5 (grain filling) and R7 (grain dry down) stages (Figure 6). Throughout our qPCR experiments, OsZIFL1, OsZIFL6, OsZIFL8, OsZIFL11 and OsZIFL13 transcripts were not detected or were detected below a confidence threshold for analysis. The expression levels of OsZIFL genes varied considerably, with some genes reaching higher expression levels (OsZIFL2 and OsZIFL4, Figures 6A and 6C) and others showing very low expression (OsZIFL3, OsZIFL9, OsZIFL5 and OsZIFL7; Figures 6B, 6D, 6E and 6G). OsZIFL2 and OsZIFL3, although not resultant of a duplication event, share a similar pattern of expression: both are more expressed in leaves and also accumulate in the later stages of flag-leaf development, reaching the highest levels in R7 (Figures 6A and 6B).

When analyzing gene pairs, we observed that OsZIFL4 is almost specifically expressed in roots, showing only little expression in panicles during the R7 stage (Figure 6C), while its duplicated copy OsZIFL9 is not expressed in vegetative tissues nor in flag-leaves, but is detected at low levels in panicles during R5 and at higher levels during R7 (Figure 6D). Transcripts from the OsZIFL5-OsZIFL10 pair show similar patterns of expression, especially when considering the reproductive organs flag-leaves and panicles (Figures $6 \mathrm{E}$ and $6 \mathrm{~F}$ ). OsZIFL5 and OsZIFL10 are both induced from R3 to R5 in flag-leaves, maintaining high levels at R7. In panicles, they are also induced from R3 to R5, although OsZIFL10 transcript levels are further induced from $\mathrm{R} 5$ to R7 (Figures $6 \mathrm{E}$ and $6 \mathrm{~F}$ ). In vegetative tissues, OsZIFL5 levels are higher in roots, while OsZIFL10 is more expressed in shoots (Figures 6E and 6F).

The genes from the OsZIFL7-OsZIFL12 pair also show similar expression patterns in the organs analyzed. OsZIFL7 is more expressed in culms and leaves, accumulates from R3 to R5 in flag-leaves and decreases its expression from R3 to R5 during panicle development (Figure 6G). The OsZIFL12 transcript accumulates in leaves and also increases from R3 to R5 in flag-leaves and decreases from R3 to R5 in panicles (Figure 6H). Taken together, our gene expression data demonstrates that, even after duplication and divergence, most OsZIFL genes still share similar expression patterns in rice organs within gene pairs.

\section{The Fe-deficiency element IDE1 is enriched in promoters of OsZIFL genes}

To investigate the presence of conserved cis-elements in promoter regions of OsZIFL genes, we used the POCO tool [53]. This approach consisted in comparing the $-1,500$ to +1 regions of OsZIFL genes to several random samples of promoters from the entire Arabidopsis genome with the same size (each sample composed of 13 promoters). If a cis-element is more often found in the promoters of OsZIFL genes than in a random set of promoters, this cis-element is enriched in these sequences. The POCO analysis revealed that the sequence CATGC is enriched in our promoter set when 


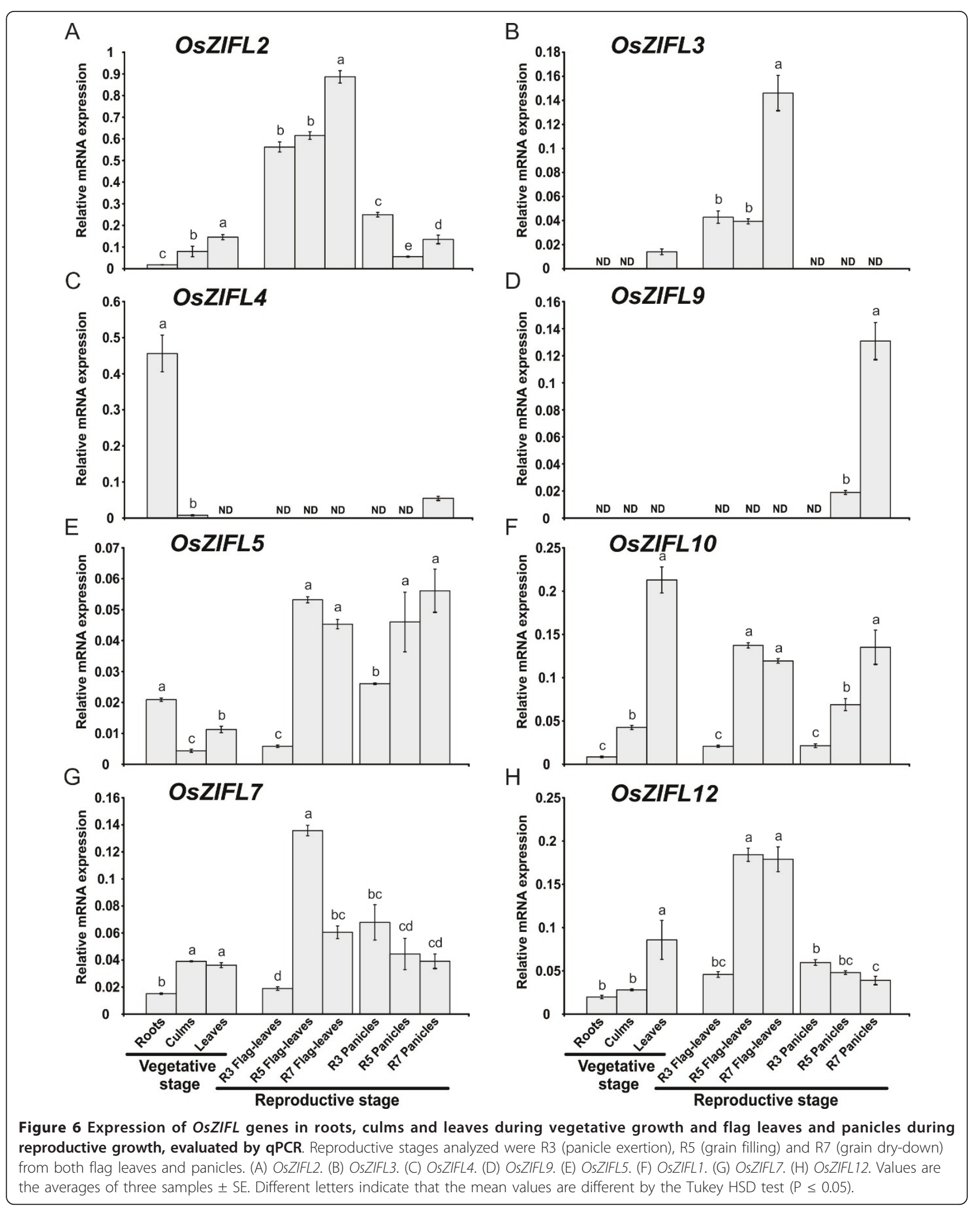




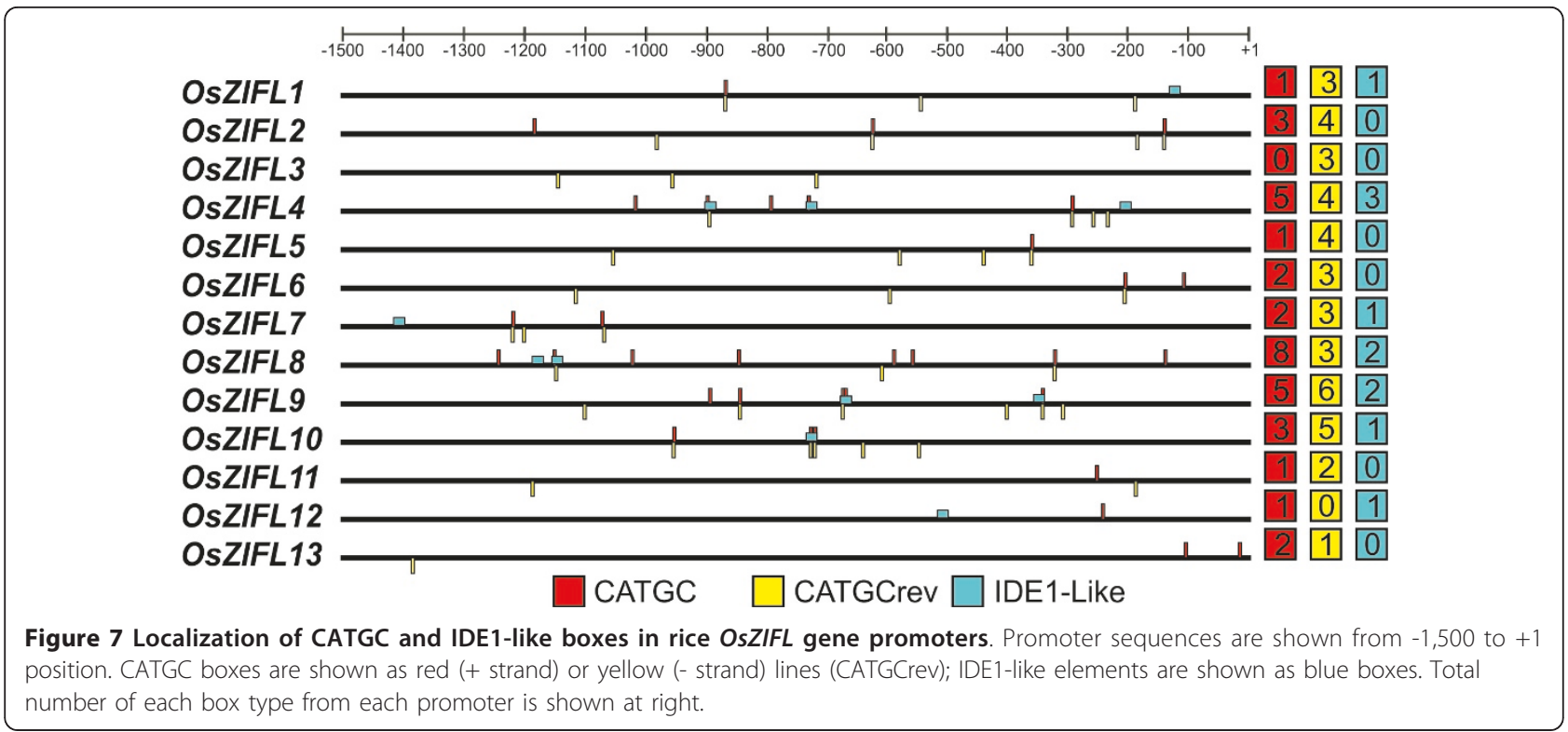

compared to Arabidopsis promoters. This sequence is the core binding site of IDEF1 (iron-deficiency responsive element-binding factor 1), a transcription factor of the ABI3/VP1 family involved in Fe-deficiency response in rice [30,54]. As Arabidopsis is not closely related to rice and thus the motif frequency in promoters could vary between these species, we confirmed the enrichment by counting the average number of CATGC boxes in nearly 25,000 promoters of rice downloaded from Osiris (http://www.bioinformatics2.wsu.edu/cgi-bin/ Osiris/cgi/home.pl) [55]. While the average number of the CATGC sequences in rice promoters was 3.24, in promoters of the thirteen OsZIFL genes it was 5.85 boxes per promoter. Some promoters are highly enriched for CATGC boxes, such as OsZIFL2 (7 boxes), OsZIFL10 (8 boxes), OsZIFL4 (9 boxes) and OsZIFL9 (10 boxes) (Figure 7). Genes that were not detected in our qPCR experiments such as OsZIFL8 and OsZIFL1 also have promoters enriched in CATGC boxes (11 and 6, respectively) (Figure 7). OsZIFL5, OsZIFL6 and OsZIFL7 promoters show 5 boxes each (Figure 7).

Since the CATGC box is the core motif of IDE1, we searched for IDE1-like sequences in promoters of OsZIFL genes following the method described by Kobayashi et al. [56]. We found eleven IDE1-like motifs distributed in seven gene promoters, OsZIFL1, OsZIFL4, OsZIFL7, OsZIFL8, OsZIFL9, OsZIFL10 and OsZIFL12 (Figure 7). OsZIFL4 shows three sequences, two of them overlapping with CATGC boxes, while OsZIFL8 and OsZIFL9 show two IDE1-like motifs (Figure 7). Considering that the motif is $18 \mathrm{bp}$ long, it is surprising to find such a high number of IDE1-like motifs in our promoter set. The enrichment for CATGC and IDE1-like sequences in promoters of OsZIFL genes suggests that they are possibly regulated by Fe-deficiency.

\section{$\mathrm{Zn}$-excess and Fe-deficiency regulate OsZIFL expression mainly in rice roots}

It has been demonstrated that AtZIF1 is up-regulated by $\mathrm{Zn}$-excess in roots and leaves of Arabidopsis plants, as well as by Fe-deficiency $[34,57,58]$. As promoters of OsZIFL genes are enriched for Fe-deficiency cis-elements, we submitted rice plants to Zn-excess $(200 \mu \mathrm{M})$ for three days and to Fe-deficiency (no Fe added to nutrient solution) for seven days. OsZIFL mRNA expression level was evaluated by $\mathrm{qPCR}$ in roots and leaves from both experiments.

Several OsZIFL genes were up-regulated in roots of $\mathrm{Zn}$ excess treated plants: OsZIFL2, OsZIFL4, OsZIFL5, OsZIFL10, OsZIFL7 and OsZIFL12 (Figure 8). Expression of OsZIFL1, OsZIFL3, OsZIFL9 and of the duplicated pairs OsZIFL6-OsZIFL11 and OsZIFL8-OsZIFL13 was not detected. Expression of OsZIFL4, which is nearly root-specific (Figure 6C), is induced 3.5 -fold by $\mathrm{Zn}$-excess (Figure 8B). Both OsZIFL5 and OsZIFL10, a duplicated pair, are also up-regulated by 2 - and 3-fold, respectively (Figures $8 \mathrm{C}$ and $8 \mathrm{D}$ ). OsZIFL7 and OsZIFL12 show different patterns of induction, with OsZIFL7 induced by almost 14-fold in comparison to control levels (Figure 8E). OsZIFL12, although induced by $\mathrm{Zn}$-excess in roots, is up-regulated only by 3 -fold (Figure $8 \mathrm{~F}$ ). To confirm that our treatment was effective, we used OsNAS1 and OsIRO2 (Figures $8 \mathrm{G}$ and $8 \mathrm{H}$ ), two genes up-regulated by $\mathrm{Zn}$-excess in rice roots [59]. Therefore, the OsZIFL genes which are expressed in roots are up-regulated under Zn-excess. 


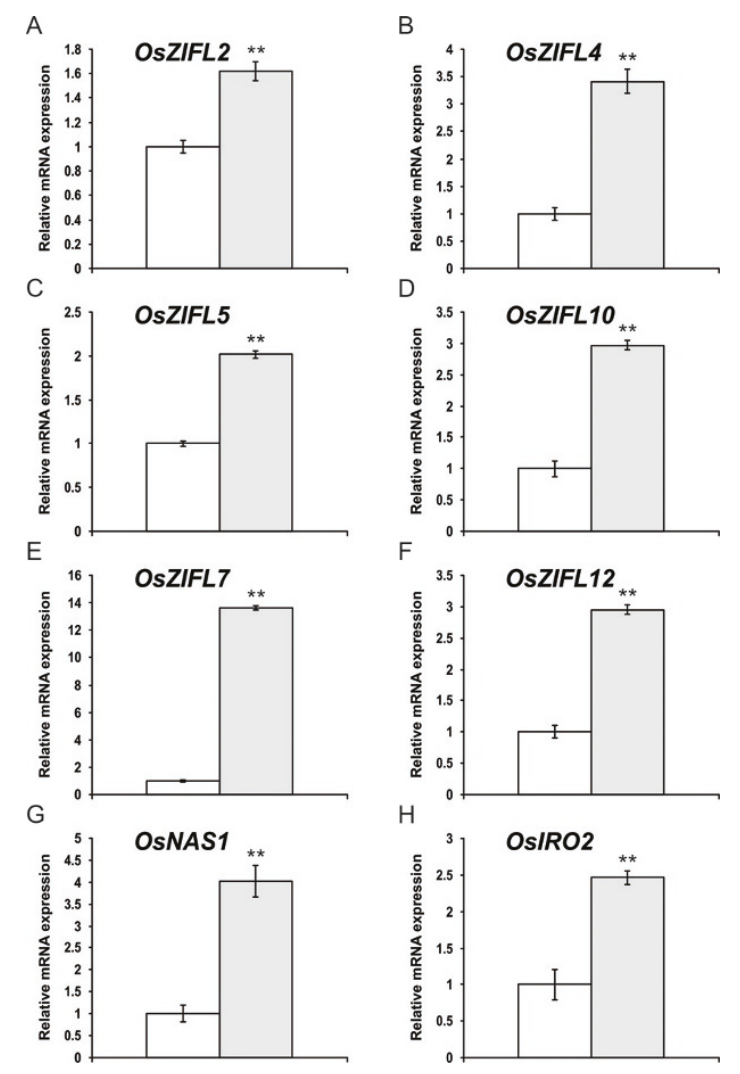

Figure 8 Gene expression in roots of rice plants submitted for 3 days to $0.5 \mu \mathrm{M}$ of $\mathrm{Zn}$ (control, white bars) or $200 \mu \mathrm{M}$ of $\mathrm{Zn}$ $(\mathrm{Zn}+$, gray bars), evaluated by qPCR. (A) OsZIFL2. (B) OsZIFL4. (C) OsZIFL5. (D) OsZIFL 10. (E) OsZIFL7. (F) OsZIFL12. (G) OsNAS1. (H) OsIRO2. Values are the averages of three samples \pm SE. Statistical differences according to the Student's t-test in comparison to control are shown by one $(p \leq 0.05)$ or two asterisks $(p \leq 0.01)$.

A very different expression pattern of OsZIFL genes was observed in leaves under $Z n$-excess: expression of OsZIFL2, OsZIFL3, OsZIFL5 and OsZIFL10 was not affected (Figures 9A, 9B, 9C and 9D). Only OsZIFL7 and OsZIFL12 mRNA levels were altered under Zn-excess: 1.4-fold and 3.3-fold higher than in the control treatment, respectively (Figures 9E and 9F). The OsZIFL7 and OsZIFL12 genes are a duplicated pair and are also upregulated by $\mathrm{Zn}$-excess in roots, suggesting a strong co-regulation under these conditions in both organs. However, most OsZIFL genes seem to be differentially regulated in leaves compared to roots when plants are under excessive $\mathrm{Zn}$ concentrations.

OsZIFL expression was also regulated in roots of plants under Fe-deficiency. Expression of OsZIFL2 and OsZIFL10 was not significantly increased by the treatment (Figures 10A and 10D). OsZIFL4, OsZIFL5, OsZIFL7 and OsZIFL12, however, were up-regulated by 1.8 to 2 -fold (Figures 10B, 10C, 10E and 10F). This

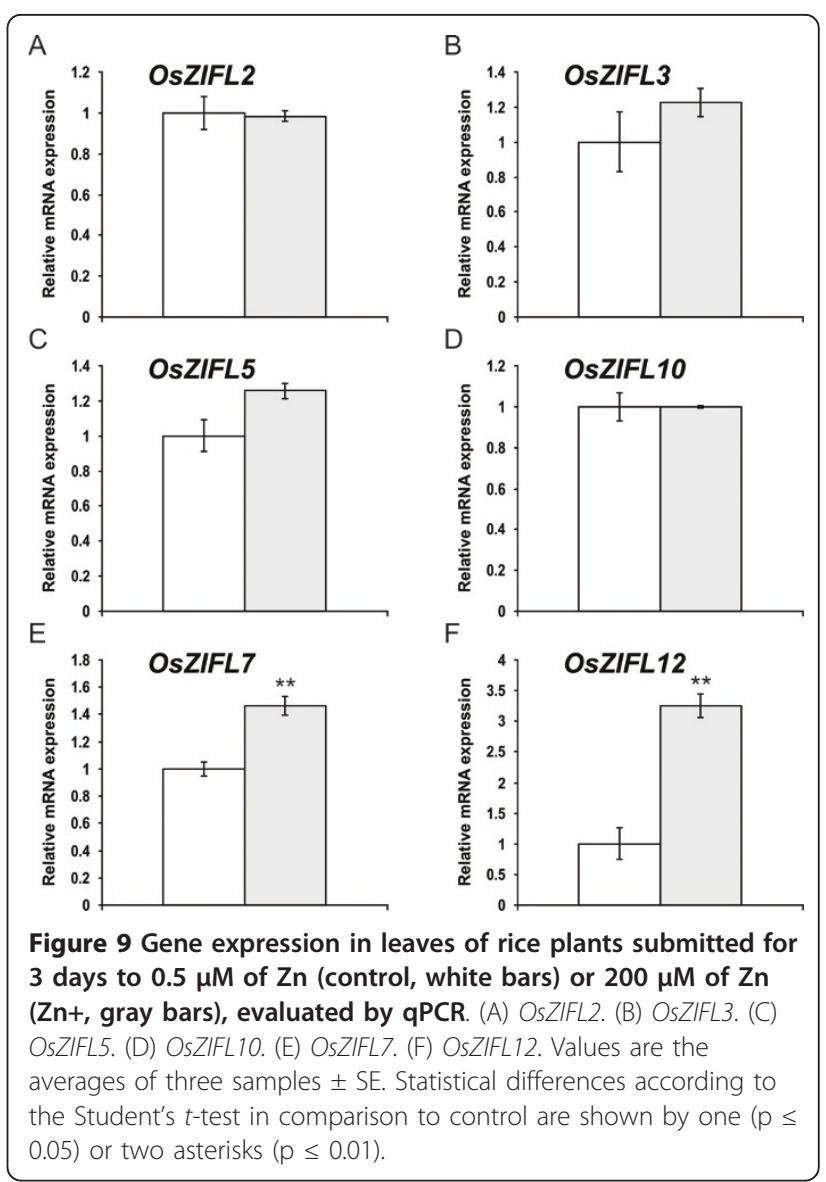

effect occurred in parallel with increased expression of OsIRT1 (2.8-fold), a gene already described as responsive to Fe-deficiency in rice roots $[60,61]$. This demonstrates that the plants were indeed under Fe-deficient conditions. Moreover, all four genes regulated by $\mathrm{Fe}$ deficiency in roots were also induced by Zn-excess (Figure 8), confirming a trend for common responses to both stresses in this organ, as previously reported [59].

A completely different response to Fe-deficiency was observed in leaves. None of the OsZIFL genes showed upregulation under this condition (Figure 11A-F), although expression of the OsIRO2 gene, was up-regulated by 5.6-fold (Figure 11G). It is known that OsIRO2 is induced by Fe-deficiency in leaves [62]. This is, however, similar to OsZIFL gene expression in leaves of Zn-excess-treated plants (Figure 9): although six OsZIFL genes were expressed, only OsZIFL7 and OsZIFL12 were up-regulated, while all other family members did not change their expression levels. Considering the results obtained with $\mathrm{Zn}$-excess and Fe-deficiency, it is possible to suggest that transcriptional regulation of most OsZIFL genes is more important in roots than in leaves, regardless of the level of expression in control conditions. 


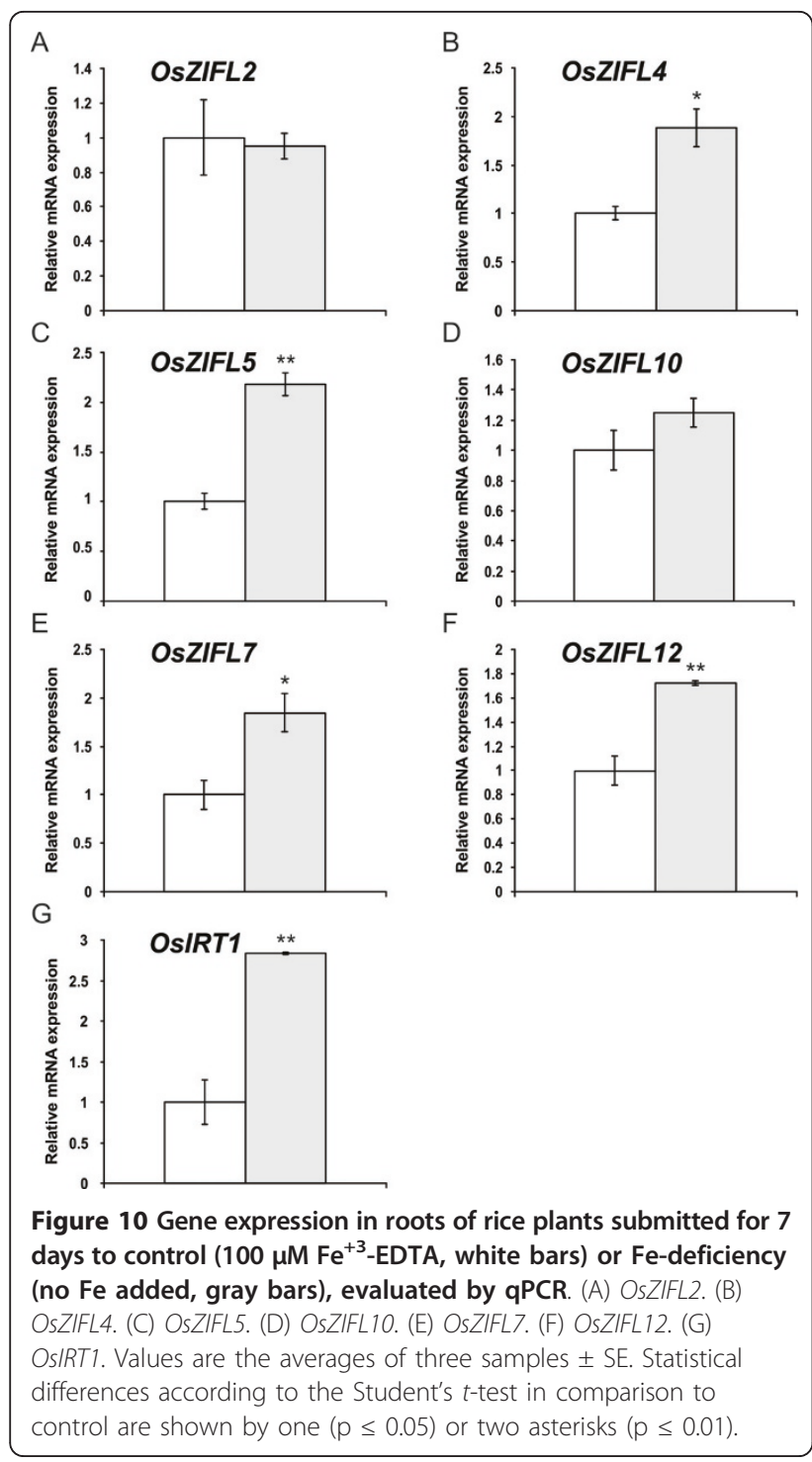

OsZIFL duplicated pairs are co-expressed in specific plant organs and in response to stresses

To analyze the expression pattern of OsZIFL genes based on microarray meta-analysis, we used Genevestigator [63]. Affymetrix unique probes used for expression analyses of OsZIFL2, OsZIFL3, OsZIFL5, OsZIFL7, OsZIFL8, OsZIFL10, OsZIFL12 and OsZIFL13 are listed in Additional File 7. The available data on expression of OsZIFL genes in different organs of rice plants is shown in Figure 12. Clearly, the expression pattern within each one of the duplicated gene pairs OsZIFL5-OsZIFL10 and OsZIFL7-OsZIFL12 cluster together, indicating their overlapping expression. According to microarray data, OsZIFL5 and OsZIFL10 are highly expressed in seed tissues, while OsZIFL7 and OsZIFL12 are expressed in reproductive organs and shoot tissues (Figure 12). Similarly, our qPCR experiments showed higher expression

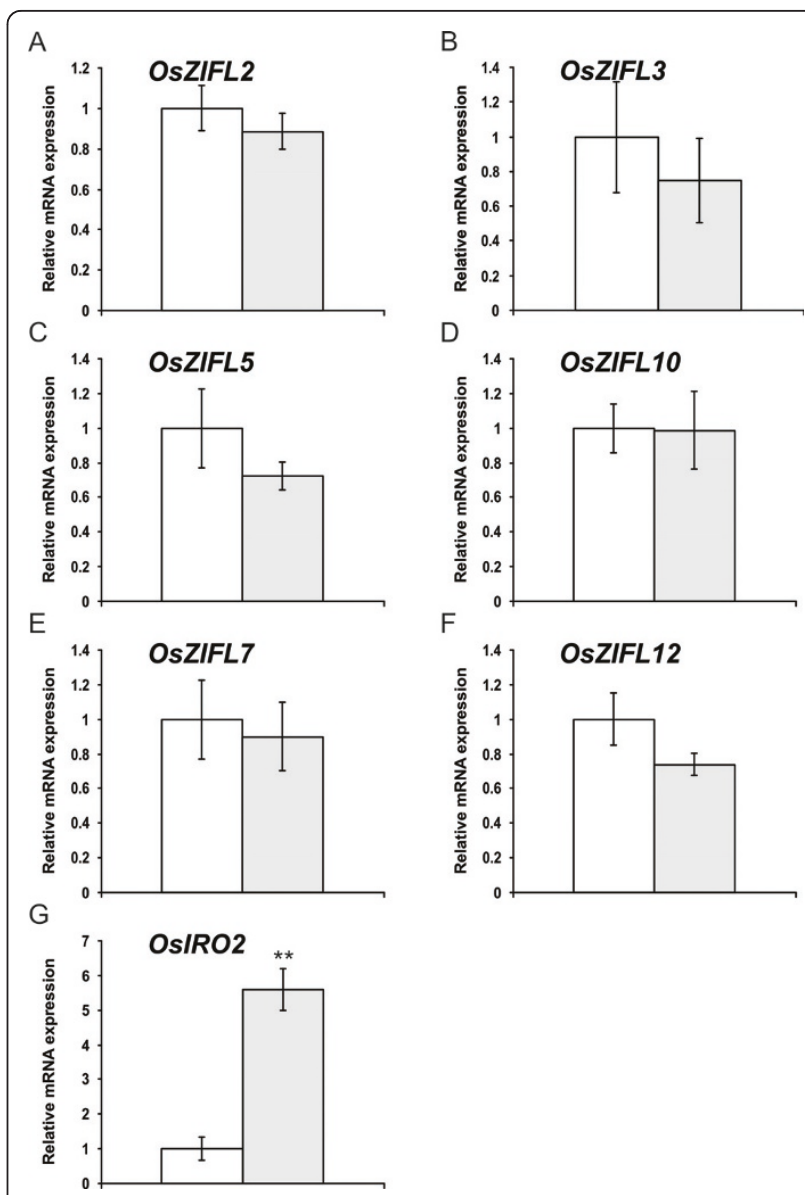

Figure 11 Gene expression in leaves of rice plants submitted for 7 days to control (100 $\mu \mathrm{M} \mathrm{Fe}^{+3}$-EDTA, white bars) or Fe-deficiency (no Fe added, gray bars), evaluated by qPCR. (A) OsZIFL2. (B) OsZIFL3. (C) OsZIFL5. (D) OsZIFL10. (E) OsZIFL7. (F) OsZIFL12. (G) OsIRO2. Values are the averages of three samples \pm SE. Statistical differences according to the Student's $t$-test in comparison to control are shown by one $(p \leq 0.05)$ or two asterisks ( $p \leq 0.01)$.

of both OsZIFL7 and OsZIFL12 in flag leaves and panicles and lower in roots (Figures 6G and 6H). The pair OsZIFL8 and OsZIFL13, which had no detected expression in our qPCR experiments, was evaluated using specific probes. While OsZIFL13 showed no expression, low expression of OsZIFL8 was observed in shoot tissues. Although qPCR will never generate the large amount of data that is achieved by cDNA microarrays, PCR has the advantage of unparalleled sensitivity, and therefore slight discrepancies are expected [64].

OsZIFL duplicated pairs also show co-expression under stress conditions (Figure 13). OsZIFL7 and OsZIFL12 are highly up-regulated by arsenate in roots of an arsenatetolerant (Bala) and an arsenate-sensitive (Azucena) cultivars (Figure 13) [65]. This suggests that these transporters could be responsive to general stress, as they are also up-regulated by Zn-excess and Fe-deficiency (Figures $8 \mathrm{E}$ 


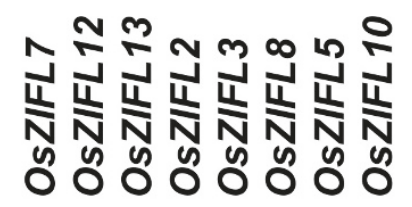

seedling coleoptile inflorescence panicle spikelet rachilla floret stamen anther pistil stigma ovary seed embryo shoot culm leaf crown rhizome roots root tip

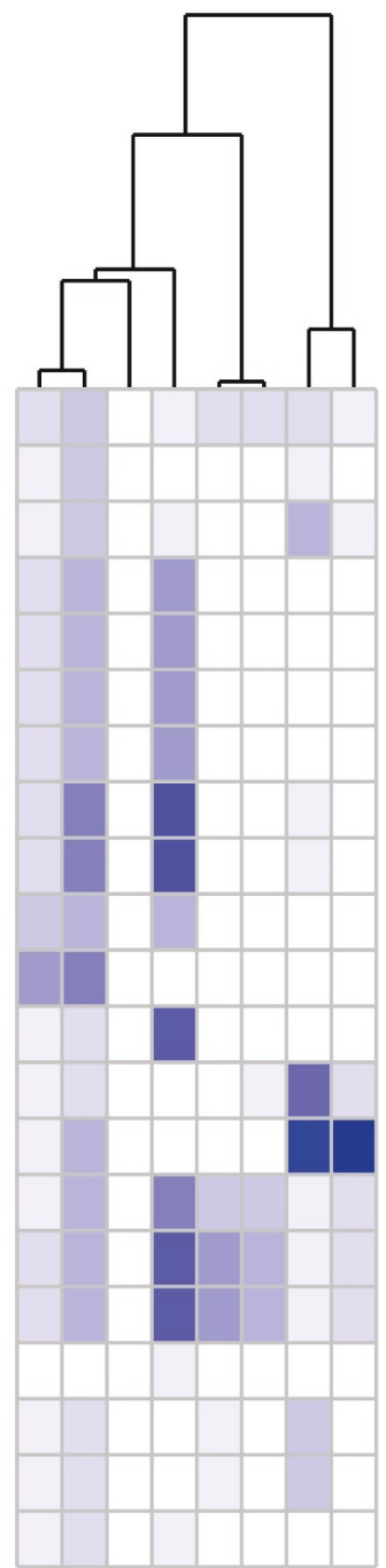

Figure 12 OsZIFL2, OsZIFL3, OsZIFL5, OsZIFL7 OsZIFL10, OsZIFL12 and OsZIFL13 gene expression data obtained using Genevestigator, and based on Affymetrix specific probes. All available high quality arrays on rice organ-specific expression were used. All data from arrays showing expression under diverse treatments or from mutant/transgenic plants were kept out. Expression level is denoted by intensity of blue color. Organ names are given at left.

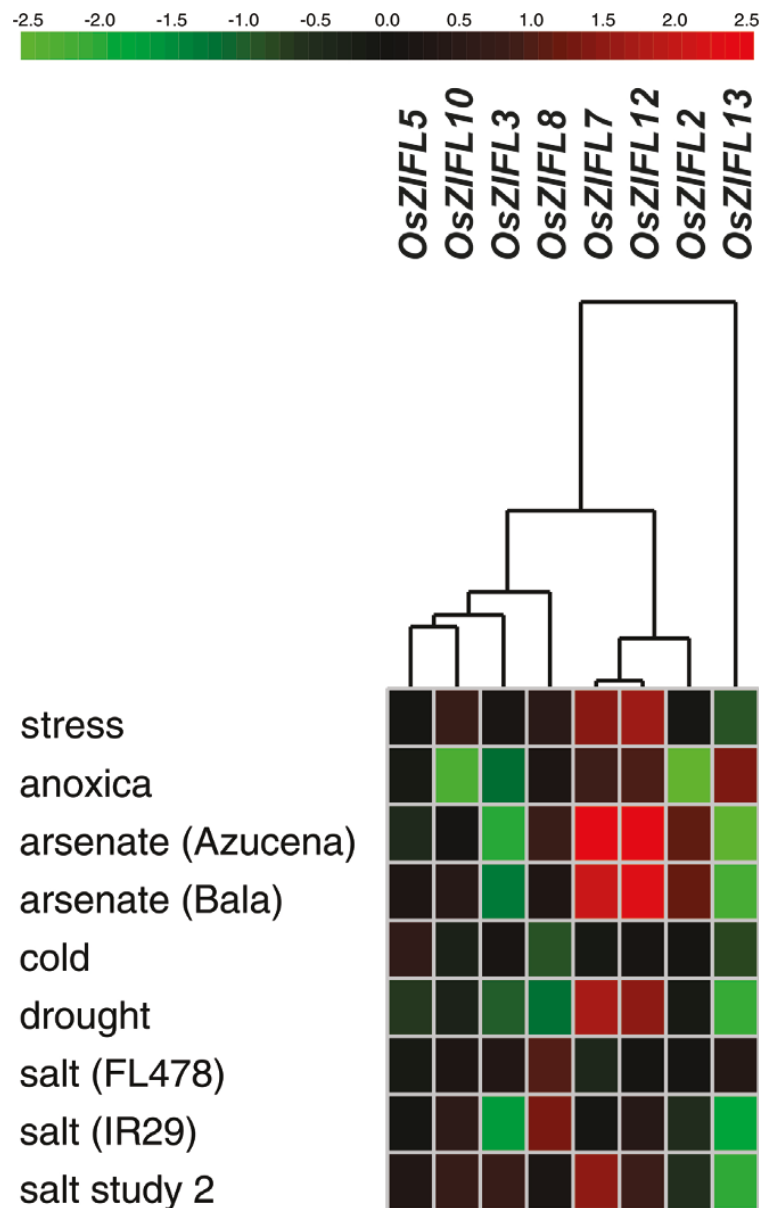

Figure 13 OsZIFL2, OsZIFL3, OsZIFL5, OsZIFL7 OsZIFL10, OsZIFL12 and OsZIFL13 gene expression data obtained using Genevestigator, and based on Affymetrix specific probes. Only high quality arrays of rice expression under diverse treatments were used. Fold change in expression level is denoted by intensity of red color (for up-regulation) or green color (for down-regulation).

Treatment names are given at left. The genotypes indicated between brackets (Azucena, Bala, FL478 and IR29) are considered, respectively, sensitive to arsenate, tolerant to arsenate, tolerant to salt and sensitive to salt.

and $8 \mathrm{~F}$; 9E and 9F, 10E and 10F). OsZIFL2 is also responsive to arsenate (Figure 13). OsZIFL7 and OsZIFL12 are also up-regulated under drought and salt stresses (Figure 13). OsZIFL5 and OsZIFL10 are mostly co-expressed, although no marked increase or decrease in expression was observed for both genes (Figure 13). The microarray results indicate a strong co-expression of the recently duplicated gene pairs OsZIFL7-OsZIFL12 and OsZIFL5OsZIFL10, in accordance with our qPCR data.

\section{Discussion}

ZIFL expansion through segmental duplication

Phenotypic variation is not necessarily the result of entirely new genes. Instead, redundancy generated through 
gene duplication can be the source of evolutionary novelty. Plants are highly susceptible to duplication events, as most (if not all) have experienced whole-genome duplication events in their evolutionary past, as well as tandem and segmental duplications [66,67]. After duplication, gene copies can follow (1) neofunctionalization, where one copy maintains the ancestral function and the other can explore new evolutionary terrain; (2) pseudogenization, where one copy accumulates mutations and lose function while the other maintains the ancestral function; (3) subfunctionalization, in which deleterious mutations make one copy to be partially functional, but complementary to the other (i. e. in regard to the ancestral gene) $[67,68]$. As deleterious mutations are expected to be more common than beneficial ones, subfunctionalization is considered to be a more common fate for duplicated copies than neofunctionalization, and examples are already known $[69,70]$. These mutations are also more common in regulatory regions (i. e. promoters) than in functional motifs, where selective pressure is stronger; therefore, changes in expression patterns and/or changes in the responses to stimuli are probably more frequent [68].

In this work, we described the ZIFL protein family in plants, which is part of the MFS superfamily. We suggested that ZIFL proteins experienced an expansion in the monocot lineage, as we found three to four gene copies in dicots and eight up to thirteen in monocots, with all monocot paralogs grouping together (Figure 2). We further characterized the genomic organization of ZIFL genes in rice, and found that ten out of thirteen copies are located in a duplicated region of chromosomes 11 and 12 (Figure 3A). This region was first described as a recent segmental duplication, estimated from five to seven MYA $[11,18]$. This estimation was based on the high degree of similarity between terminal segments of both chromosomes (Figure 3). However, recent data showed that the duplication of this genomic segment is ancestral to the split of S. bicolor, B. distachyon and rice $[14,19]$. Wang et al. proposed that three rounds of unequal crossing-over events have produced the high similarity observed [9]. Thus, variation in sequence similarity within these regions reflects rather the antiquity of the unequal crossing-over events, than the date of segmental duplication as suggested earlier $[11,18]$. Gene conversion is also occurring at high frequencies within this region, further contributing for the maintenance of high similarity $[9,13,15]$. Using paralog pairs within the $3 \mathrm{Mb}$ of chromosomes 11 and 12 from all species in the Oryza genus, a recent work demonstrated that concerted evolution is recurrent in this region for Oryza species [19]. Gene conversion was specifically found between OsZIFL4 and OsZIFL9 in indica rice, suggesting that concerted evolution has participated in the evolution of ZIFL genes [9].
We also demonstrated that the region where S. bicolor ZIFL genes are located in chromosome 8 is inverted in relation to its homologous region in rice chromosome 12 (Figures $3 \mathrm{~B}$ and $3 \mathrm{C}$ ). This inverted region was recently described for both $S$. bicolor and B. distachyon, encompassing $0.8 \mathrm{Mb}$ [19]. S. bicolor ZIFL gene pairs are not as similar as rice paralogs, indicating that $S$. bicolor ZIFL genes probably did not undergo the same degree of concerted evolution as rice paralogs (i.e. unequal crossing-over and gene conversion). In agreement with that, Wang et al. used paralog quartets from rice and S. bicolor (i.e. a duplicated gene pair from rice and their homologs from $S$. bicolor) to search for gene conversions. They found that OsZIFL4 and OsZIFL9 went through whole gene conversion after the split between rice and S. bicolor, while S. bicolor homologs did not show conversion (in their supplemental table) [15]. Inversions are known to reduce the probability of recombination and to facilitate the maintenance of differences between interbreeding populations $[71,72]$. These results suggest that the inversion observed in $S$. bicolor reduced the probability of concerted evolution in the SbZIFL genes when compared to rice paralogs.

\section{Sequence and expression analyses suggest new functional sites in OsZIFL proteins and insights about duplicated gene pairs}

Our analysis on motif composition of OsZIFL proteins also revealed interesting features of this family in rice. Together with the exon-intron organization (Figure 4), motif composition of duplicated genes OsZIFL8 and OsZIFL13 and their partial duplicated copy OsZIFL3 suggests that these genes are diverging in a higher rate when compared to other OsZIFL paralogs. They all show no ZIFL signature Cys motif C-P-G-C (Additional File 4). OsZIFL3 and OsZIFL8 also lack the MFS signature and OsZIFL13 lacks both MFS and antiporter signatures (Additional File 4). OsZIFL3 expression was detected in leaves, but at relatively low levels (Figure 6B). OsZIFL8 and OsZIFL13 transcripts were not detected in any of our qPCR experiments, and cDNAs corresponding to them are not present at the KOME database (http://cdna01.dna.affrc.go.jp/PIPE/). However, microarray metadata showed low expression of OsZIFL8 in shoots, although no expression of OsZIFL13 was detected in all plant organs evaluated (Figure 12). Further experiments should clarify if these genes are gaining new functions or accumulating mutations to become pseudogenes.

A variable region, which corresponds to a cytoplasmic loop, occupies a central position in the OsZIFL proteins (Figures 1B, 5A and Additional File 6). There is very low amino acid conservation within this loop. For this reason, we were able to find conserved motifs within the variable region only when using the whole ZIFL protein 
dataset in our analyses (Figure 5B). Variable regions are often found in transporters [50,51]. In the ZIP family (Zinc-regulated/Iron-regulated transporter Proteins), a variable region is considered to be the metal-binding site, as these loops are rich in histidine residues [50,51]. Our motif analysis in the ZIFL variable region detected some residues in conserved positions. In the CDF family, substrate specificity was proposed to be determined by few amino acids, normally histidine $(\mathrm{H})$ or aspartic acid (D), which are, respectively, positively and negatively charged [52]. In OsZIFL proteins, lysine (K) and glutamic acid $(\mathrm{E})$, also positive and negative residues, seem to be conserved in the variable loop (Figure 4B), although aspartic acid (D) and leucine (L) are also frequent (Figure 5B). This region and its conserved residues emerge as candidates for mutagenesis studies to clarify their importance in substrate transport, although no substrate was proven to be transported by ZIFL proteins [34]. Moreover, we described conserved motifs specific to ZIFL proteins (Figure 1B), which also contain candidate residues for site-directed mutagenesis studies.

We characterized the expression of OsZIFL genes in rice vegetative and reproductive organs (Figure 6) and compared the expression patterns of three duplicated gene pairs, OsZIFL4-OsZIFL9, OsZIFL5-OsZIFL10 and OsZIFL7-OsZIFL12 (Figure 6). OsZIFL4 and OsZIFL 9 are both expressed in panicles at R7 stage, but only OsZIFL4 is expressed in roots (Figures 6C and 6D). This partial overlap suggests that their ancestral gene was at least expressed in panicles at R7 and in roots, as deleterious mutations could be subfunctionalizing OsZIFL9 (i.e. turning into a panicle-specific gene) while OsZIFL4 maintains both panicle and root expression. However, neofunctionalization of OsZIFL4 cannot be discarded. In agreement with that, Throude et al. showed that, from 115 duplicated gene pairs, the vast majority have been neofunctionalized or subfunctionalized, as $88 \%, 89 \%$ and $96 \%$ of duplicates, respectively expressed in grain, leaf and root, show distinct expression patterns [73]. A recent work in rice showed that the average number of conserved motifs between duplicated gene pairs declines with increased expression diversity, partially supporting the subfunctionalization model [74]. This is in accordance with the observed divergent expression of OsZIFL4 and OsZIFL9 and their motif composition, because OsZIFL9 has lost one $\mathrm{N}$-terminal and two C-terminal motifs (Figure 5A). Expression patterns within the gene pairs OsZIFL5OsZIFL10 and OsZIFL7-OsZIFL12 are similar (Figure 6), and both OsZIFL5 and OsZIFL10 have the same motif composition (Figure 5A). OsZIFL12 has two C-terminal motifs which are lacking in OsZIFL7 (Figure 5A), but expression of OsZIFL7 and OsZIFL12 is quite similar, as both are up-regulated in roots and leaves under Zn-excess (Figures 8E, 8F, 9E and 9F) and in roots under Fe-deficiency (Figures 10E and 10F). Microarray data also shows that the OsZIFL duplicated pairs OsZIFL5-OsZIFL10 and OsZIFL7-OsZIFL12 are coexpressed in the same plant organs and under the same treatments (Figures 12 and 13). Yim et al. showed that duplicated gene pairs with high local similarity (HLS) segments show higher expression correlations than gene pairs without these segments [74]. This probably results in an increased likelihood of gene conversion in promoters of gene pairs harboring HLS [74]. As gene conversion is known to homogenize sequences in multigene families, this probably explains the similar expression patterns of OsZIFL pairs, although it is established that duplicated gene pairs tend to rapidly diverge in their expression patterns $[12,13,75]$.

\section{Expression of OsZIFL genes is involved in the partially overlapping pathways of $\mathrm{Zn}$-excess and Fe-deficiency responses}

Ten out of thirteen OsZIFL genes are found in two tandem groups of five genes in rice chromosomes 11 and 12 , probably as a result of repeatedly tandem duplication events. This size of tandemly arrayed genes was estimated to be very rare, as only $7 \%$ of gene arrays in the rice genome have more than three genes [76]. Tandem duplication events have a tendency to be retained when involving genes for which fluctuation in copy number is unlikely to affect downstream genes, such as those at the end of or in flexible steps of pathways [76]. In Arabidopsis and rice, tandemly arrayed genes are enriched for membrane proteins and genes with function on abiotic and biotic stresses [76]. Moreover, tandemly arrayed genes often share regulatory cis-elements and tend to be expressed in a coordinated manner, as well as family members with HLS generated through gene conversion [74,77]. These observations are in accordance with the up-regulation of OsZIFL members under $\mathrm{Zn}$-excess or Fe-deficiency, some of which show strong up-regulation upon stress imposition, mostly in roots (Figures 8, 9, 10 and 11). It also agrees with the enrichment observed for CATGC and IDE1-like elements in OsZIFL promoter sequences (Figure 7). Enrichment for the CATGC-box is related to Fe-deficiency responses in rice $[30,78]$. The rice specific gene OsMIR is strongly up-regulated by Fe-deficiency and shows 10 CATGCboxes in its promoter sequence [78]. In another work, CATGC was shown to be enriched in promoters of genes regulated by OsIDEF1, an upstream transcription factor involved in the early response to Fe-deficiency [30]. Thus, OsZIFL genes which are responsive to Fedeficiency are potentially under the same control network, although more data is necessary to confirm this 
hypothesis. Moreover, a similar up-regulation pattern is also observed in the Arabidopsis AtZIF1 gene, which is also responsive to both $\mathrm{Zn}$-excess and Fe-deficiency $[34,57,58]$. This suggests that OsZIFL genes which are responsive to both stresses could have conserved regulatory sequences in comparison to AtZIF1.

Partial overlap between $\mathrm{Zn}$-excess and Fe-deficiency response has been reported [59]. Zn-excess treated plants show much higher concentrations of Fe in roots, but slightly decreased Fe in shoots and inhibited expression of OsFER1 [59]. This indicates that $\mathrm{Zn}$-excess causes Fe-deficiency due to mislocalization of the available Fe [59]. On the other hand, Fe-deficiency can cause $\mathrm{Zn}$-excess, as $\mathrm{Fe}$ regulated transporters such as OsIRT1 are suggested to transport $\mathrm{Zn}$ and Arabidopsis plants under Fe-deficiency accumulate excessive $\mathrm{Zn}$ $[39,79]$. It was also demonstrated that $13.75 \%$ of the $\mathrm{Zn}$ excess up-regulated genes in roots are also up-regulated by Fe-deficiency, further indicating an overlap between these stresses [59]. Excessive Zn was also shown to induce more genes in rice roots than in shoots, as 400 genes were induced in roots, while only 54 in shoots of Arabidopsis plants under Zn-excess [59].

OsIRO2, a bHLH (basic Helix-Loop-Helix) transcription factor induced by Fe-deficiency, is the regulator of Fe-deficiency responsive genes in roots, such as the genes OsNAS1 (nicotianamine synthase 1), OsNAS2, OsNAAT1 (nicotianamine amino-transferase 1), OsDMAS1 (deoxymugineic acid synthase 1) and the DMA-Fe ${ }^{3+}$ transporter OsYSL15 [80]. Expression of OsIRO2 was shown to be up-regulated by and to control the induction of these genes under $\mathrm{Zn}$-excess [59]. However, OsIRT1, a classical Fe-deficiency-regulated gene, is not regulated by OsIRO2 [80], and is not upregulated under $\mathrm{Zn}$-excess [59]. These results indicate that OsIRO2 is in the crosslink between $\mathrm{Zn}$-excess and Fe-deficiency responses. The OsIRO2 binding site CACGTGG is not found in OsZIFL promoters, but our qPCR data shows that OsZIFL4, OsZIFL5, OsZIFL7 and OsZIFL12 are up-regulated in roots by both stresses (Figures 8 and 10). Considering our results, it is possible to suggest that OsZIFL genes are part of the overlapping pathway that links Fe-deficiency and $\mathrm{Zn}$-excess, although regulators different from OsIRO2 may control their expression. One of these regulators could be IDEF1 [54].

\section{Conclusions}

As the first description of the ZIFL family in plants, this work is the basis for functional studies, especially in rice. We have shed light onto the unusual genomic distribution of OsZIFL genes, and made suggestions about the evolutionary forces that shaped the high degree of similarity between them. We also characterized in detail the motif composition of rice OsZIFL genes and the expression patterns in different rice organs and under stress conditions. More functional data, such as loss-offunction mutants, sub cellular localization and ligand specificity, are necessary to uncover the specific roles of each protein and to know to what extent they are functionally redundant, as well as to clarify the roles of OsZIFL genes in the homeostasis of $\mathrm{Zn}$ and Fe in rice.

\section{Methods}

\section{Plant material and treatments}

Rice seeds of the Nipponbare cultivar were germinated for four days in petri dishes, soaked in distilled water at $28^{\circ} \mathrm{C}$ (two days in the dark, two days in the light). After germination, seedlings were transferred to holders positioned over plastic pots with five liters of nutrient solution (16 seedlings per pot) containing $700 \mu \mathrm{M} \mathrm{K}_{2} \mathrm{SO}_{4}$, $100 \mu \mathrm{M} \mathrm{KCl}, 100 \mu \mathrm{M} \mathrm{KH_{2 }} \mathrm{PO}_{4}, 2 \mathrm{mM} \mathrm{Ca}\left(\mathrm{NO}_{3}\right)_{2}$, $500 \mu \mathrm{M} \mathrm{MgSO}_{4}, 10 \mu \mathrm{M} \mathrm{H}_{3} \mathrm{BO}_{3}, 0.5 \mu \mathrm{M} \mathrm{MnSO}_{4}, 0.5 \mu \mathrm{M}$ $\mathrm{ZnSO}_{4}, 0.2 \mu \mathrm{M} \mathrm{CuSO}_{4}, 0.01 \mu \mathrm{M}\left(\mathrm{NH}_{4}\right)_{6} \mathrm{Mo}_{7} \mathrm{O}_{24}$, and $100 \mu \mathrm{M} \mathrm{Fe}^{+3}$-EDTA. The $\mathrm{pH}$ of the nutrient solution was adjusted to 5.4. Plants were kept at $28^{\circ} \mathrm{C} \pm 1^{\circ} \mathrm{C}$ under photoperiod of $16 \mathrm{~h} / 8 \mathrm{~h}$ light/dark $\left(150 \mu \mathrm{mol} . \mathrm{m}^{-2} . \mathrm{s}^{-}\right.$ $\left.{ }^{1}\right)$. Solutions were replaced every 3-4 days.

For expression analyses in vegetative organs, samples of roots, leaves and culms were collected at the four-leaf stage (approximately 30 days of growth). For expression analyses in reproductive organs, plants were grown in soil under flooded conditions in an experimental unit at IRGA (Instituto Rio-Grandense do Arroz), in Cachoeir-

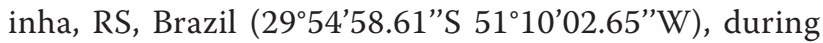
the rice growing season (October 2007 to March 2008). Soil characteristics of this site were reported by Stein et al. [36]. Samples of flag-leaves and panicles were collected during R3 (panicle exertion), R5 (grain filling) and R7 (grain dry-down) stages, according to Counce et al. [81]. Laboratory grown plants at the four-leaf stage were submitted to $\mathrm{Zn}$-excess or to Fe-deficiency. For $\mathrm{Zn}$-excess, plants were kept in $200 \mu \mathrm{M}$ of $\mathrm{ZnSO}_{4}$ for 3 days. For Fe-deficiency, $\mathrm{Fe}^{+3}$-EDTA was omitted from nutrient solution and samples were collected after 7 days. In all experiments, three biological samples composed of at least three plants each were used for gene expression analyses.

\section{Sequence retrieval and databases}

Sequences of Arabidopsis thaliana AtZIF1 (AT5G13740), AtZIFL1 (AT5G13750) and AtZIFL2 (AT3G43790) proteins were downloaded from the TAIR database (The Arabidopsis Information Resource, http:// www.arabidopsis.org/) and used as queries to search the rice genome at the TIGR Rice Genome database (http:// rice.plantbiology.msu.edu/) for ZIFL sequences using tBLASTn and BLASTp. Sequences with an expected 
value lower than $1 \times 10^{-30}$ and harboring more than $30 \%$ of similarity considering $30 \%$ of the sequence were selected. Both Arabidopsis and rice sequences were then used as queries to survey the genomes of Vitis vinifera, Populus trichocarpa, Sorghum bicolor, Brachypodium distachyon, Zea mays, Selaginella moellendorffii and Physcomitrella patens at the Phytozome database (http://www.phytozome.net/), using the same criteria as above. All plant sequences found (plus the previously known Arabidopsis sequences) were aligned and used as an input to build an HMM profile using the HMMER package [82]. The ZIFL HMM profile consensus sequence was used to re-search the listed genomes. As new sequences were found, the procedure was repeated iteratively until no new sequence appeared. To visualize the ZIFL HMM profile and the conserved motifs we used LogoMat M [83]. Alignments of ZIFL profile to MFS_1 HMM profile were performed using LogoMat P [84]. Individual sequences were manually curated to discard those of poor quality or incomplete (not starting with methionine or not having a stop codon). Accession numbers, given nomenclature, chromosome and genomic positions and predicted number of transmembrane domains (TMs) of ZIF-like proteins are shown in Additional File 1. Two unnanotated ZIFL genes from Zea mays were predicted using Fgenesh (http://www.softberry.com) and their given nomenclature, chromosome and genomic positions, exon coordinates and predicted number of TMs are shown in Additional File 2.

Sequences from MFS_1 proteins from each monocot and dicot species analyzed in this work were retrieved using the same method as for ZIFL protein sequences. The consensus sequence generated from the HMM profile of MFS_1 (Pfam number PF07690) was used to search the genomes. All locus numbers of MFS_1 genes used are given in Additional File 8.

\section{Alignments and phylogenetic analyses}

Sequence alignment and phylogenetic analyses for ZIFL proteins were performed using the MEGA (Molecular Evolutionary Genetics Analysis) 4.1 package [85]. Protein multiple alignments were obtained with ClustalW and phylogenetic trees were reconstructed with the neighbor-joining method and the following parameteres: pairwise deletion option, 1,000 replicates of bootstrap and Poisson correction distance. The consensus tree shows only branches with a bootstrap consensus $>50$. Bayesian analysis was applied to generate a posterior probability distribution using the Metropolis-coupled Markov Chain Monte Carlo (MCMC) with MrBayes 3.0b4 [86,87]. The search was run for $1 \times 10^{6}$ generations, and every 100th tree was sampled. Posterior probabilities for each branch were calculated from the sampled trees. Sequence alignments of MFS_1 proteins were performed using MEGA 4.1 package, and phylogenetic trees were reconstructed with the neighbor-joining method, following the same parameters described above.

For genomic alignments, we used a $100 \mathrm{~kb}$ region from rice chromosomes 11 and 12 and S. bicolor chromosomes 5 and 8 spanning the tandemly repeated ZIFL genes regions in both species. The graphic alignment tool for comparative sequence analysis (GATA) was used to align the sequences and visualize the results [88]. GATA uses BLASTn to compare a reference to a comparative sequence. A sliding window of predefined size slides through the reference sequence and aligns it to the comparative sequence. Matches are shown in black for forward hits $(+/+)$ and in red for reverse hits $(+/-)$. We used a window size of 24 and a lower cutoff score of 80 . The default values were used for the other settings.

\section{Exon-intron determination, motif finding and promoter analysis}

For determining exon-intron organization, genomic and coding sequences (predicted, cDNA when available) were aligned. To search for conserved motifs in ZIF-like proteins, MEME (Motif EM for Motif Elicitation - http:// meme.nbcr.net/ [49]) was used, with the following parameters: zero or one motif per sequence, 6 and 300 amino acids as minimum and maximum sizes of motifs. Only motifs with expected value lower than $1 \times 10^{-20}$ were considered. For motifs within the variable region, the e-value cutoff was increased to $1 \times 10^{-10}$ due to high sequence divergence. The best possible match of each motif was searched in the InterPro database (http://www. ebi.ac.uk/interpro/). To identify the transmembrane domains of ZIFL proteins we used Conpred II (http:// bioinfo.si.hirosaki-u.ac.jp/ ConPred2/), a consensus prediction method for obtaining transmembrane topology models. Promoter sequences from $-1,500 \mathrm{bp}$ to $+1 \mathrm{bp}$ of each rice ZIF-like gene were extracted from the TIGR Rice Genome database. Different strategies were used to find regulatory sequences within the promoters of OsZIFlike genes. POCO was used to compare the promoter dataset to the Arabidopsis thaliana clean background, the closest species available for this tool [53]. POCO was run with default settings, except for the pattern length selected as $5 \mathrm{bp}$. To confirm that over-represented motifs in comparison to Arabidopsis background are also overrepresented when compared to rice background, the $-1,500$ bp to +1 bp promoter region of nearly 25,000 rice genes were downloaded from the Osiris database (http:// www.bioinformatics2.wsu.edu/cgi-bin/Osiris/cgi/home.pl/ [55]) and evaluated for average number of motifs.

\section{Genevestigator}

We used only specific Affymetrix probes for rice ZIF genes (Additional File 7) to analyze expression data 
from GENEVESTIGATOR (http://www.genevestigator. com) [63]. Only high quality arrays were used.

\section{RNA extraction and CDNA synthesis}

Rice tissues were harvested from plants grown under laboratory or field conditions as described above. Total RNA was extracted using the Concert Plant RNA Reagent (Invitrogen ${ }^{\circledR}$, Carlsbad, CA, USA) and treated with DNase I (Invitrogen ${ }^{\circledR}$, Carlsbad, CA, USA). cDNA was prepared using the SMART PCR cDNA Synthesis Kit by Clontech ${ }^{\circledR}$ Laboratories (Mountain View, CA, USA), according to the manufacturer's instructions. First-strand cDNA synthesis was performed with oligo $\mathrm{dT}$ and reverse transcriptase (M-MLV, Invitrogen ${ }^{\circledR}$, Carlsbad, CA, USA) using $1 \mu \mathrm{g}$ of RNA.

\section{Quantitative RT-PCR and data analysis}

For quantitative RT-PCR analysis (qPCR), the synthesized first strand cDNA from each time point was diluted 100 times. qPCR was carried out in an Applied Biosystems StepOne real-time cycler. All primers (listed in Additional File 9) were designed to amplify 100-150 bp of the 3'-UTR of the genes and to have similar Tm values $\left(60 \pm 2^{\circ} \mathrm{C}\right)$. Reaction settings were composed of an initial denaturation step of $5 \mathrm{~min}$ at $94^{\circ} \mathrm{C}$, followed by 40 cycles of $10 \mathrm{~s}$ at $94^{\circ} \mathrm{C}, 15 \mathrm{~s}$ at $60^{\circ} \mathrm{C}, 15 \mathrm{~s}$ at $72^{\circ} \mathrm{C}$; samples were held for $2 \mathrm{~min}$ at $60^{\circ} \mathrm{C}$ for annealing of the amplified products and then heated from 60 to $99^{\circ} \mathrm{C}$ with a ramp of $0.3^{\circ} \mathrm{C} / \mathrm{s}$ to provide the denaturing curve of the amplified products. qPCRs were carried out in $20 \mu \mathrm{l}$ final volume composed of $10 \mu \mathrm{l}$ of each reverse transcription sample diluted 100 times, $2 \mu \mathrm{l}$ of 10X PCR buffer, $1.2 \mu \mathrm{l}$ of $50 \mathrm{mM} \mathrm{MgCl}, 0.1 \mu \mathrm{l}$ of $5 \mathrm{mM} \mathrm{dNTPs}, 0.4 \mu \mathrm{l}$ of $10 \mu \mathrm{M}$ primer pairs, $4.25 \mu \mathrm{l}$ of water, $2.0 \mu \mathrm{l}$ of SYBR green (1:10,000, Molecular Probe), and $0.05 \mu \mathrm{l}$ of Platinum Taq DNA polymerase $\left(5 \mathrm{U} / \mu \mathrm{l}\right.$, Invitrogen ${ }^{\circledR}$, Carlsbad, CA, USA). Obtained data were analyzed using the comparative $C_{t}$ (threshold cycle) method [89]. The PCR efficiency from the exponential phase (E) was calculated for each individual amplification plot using the LinRegPCR software [90]. In each plate, the average of PCR efficiency for each amplicon was determined and used in further calculations. $C_{t}$ values for all genes were normalized to the $C_{t}$ value of $U B Q 5$ [91]. The equation $Q_{0}$ target gene/ $\mathrm{Q}_{0 \text { UBQ5 }}=\left[\left(\mathrm{Eff}_{\mathrm{UBQ}}\right)^{\mathrm{Ct} \mathrm{UBQ}} /\left(\mathrm{Eff}_{\text {target gene }}\right){ }^{\mathrm{Ct} \text { target gene }}\right]$, where $\mathrm{Q}_{0}$ corresponds to the initial amount of transcripts, was used for normalization [89]. Each data point corresponds to three true biological replicate samples.

\section{Statistical analyses}

When appropriate, data were subjected to ANOVA and means were compared by the Tukey HSD or Student's $t$ test using the SPSS Base 12.0 for Windows (SPSS Inc., USA).

\section{Abreviations}

CDF: cation diffusion facilitator; CDS: coding sequence; OsDMAS1: deoxymugineic acid synthase; DDC: duplication-degeneration-complementation; HLS: high local similarity; IDE1: iron-deficiency responsive element 1; IDEF1: iron-deficiency responsive element-binding factor 1; MFS: major facilitator superfamily; MYA: million years ago; NAAT1: nicotianamine amino transferase; NAS: nicotianamine synthase; ORF: open reading frame; ZIF: zinc-induced facilitator; ZIFL: zinc-induced facilitator-like; ZIP: zinc-regulated/iron-regulated transporter protein

\section{Additional material}

Additional File 1: ZIFL gene sequence information. Gene locus number, given name, chromosome number, genomic localization, strand, predicted coding sequence (CDS) and protein length, and predicted number of transmembrane domains (TM) are shown for each gene.

Additional File 2: Previously unannotated ZIFL genes. Gene locus number, given name, chromosome number, genomic localization, strand, predicted coding sequence (CDS) and protein length, exon positions and predicted number of transmembrane domains (TM) are shown for each gene.

Additional File 3: Phylogenetic trees of ZIFL and MFS_1 proteins Phylogenetic trees showing the separation of ZIFL proteins from the other MFS_1 sequences in each monocot and dicot species analyzed. (A) Oryza sativa, (B) Sorghum bicolor, (C) Zea mays, (D) Brachypodium distachyon, (E) Arabidopsis thaliana, (F) Vitis vinifera, (G) Populus trichocarpa.

Additional File 4: Conserved residues found in ZIFL protein sequences. Residues of cysteine (Cys) motif, histidine (His) motif, TM8TM9 loop motif, and residues of MFS and antiporter signatures of each ZIFL protein are shown.

Additional File 5: Chromosomal positions of ZIFL genes

Chromosomal positions of ZIFL genes in (A) Oryza sativa, Sorghum bicolor and Brachypodium distachyon chromosomes, and in (B) Zea mays chromosomes. Only ZIFL-containing chromosomes are shown. Non-ZIFL genes within ZIFL gene clusters were omitted.

Additional File 6: Alignment of OsZIFL protein sequences. Alignment was constructed using ClustalW. Conserved amino acids are marked in black or grayscale according to similarity level.

Additional File 7: Probes used in Genevestigator analyses and evaluation of specificity of rice ZIFL Affymetrix ${ }^{\mathbb{B} \otimes}$ microarray probes. For each ZIFL gene locus number, all corresponding probes are listed. Each probe is classified as unique or not unique, and, in the second case, the number of the other locus matching to the probe is provided.

Additional File 8: Locus numbers of MFS_ 1 genes used in reconstruction of phylogenetic trees, in addition to ZIFL genes. Locus numbers of MFS_l genes used in the reconstruction of phylogenetic trees shown in Additional File 3, in addition to ZIFL genes (Additional File 1 and Additional File 2).

Additional File 9: Gene-specific primers used for quantitative RTPCR. Sequences of PCR primers used in quantitative RT-PCR analyses of rice ZIFL gene expression.

\section{Acknowledgements}

This research was supported by CNPq (Conselho Nacional de Desenvolvimento Científico e Tecnológico, Brazil): research fellowship to JPF and scholarships to FKR, PKM and ERS. RAS was recipient of a scholarship 
from CAPES (Coordenaação de Aperfeiçoamento de Pessoal de Nível Superior, Brazil) and KLL from FAPERGS (Fundação de Apoio à Pesquisa do Rio Grande do Sul). The authors thank IRGA (Instituto Rio-Grandense do Arroz) for technical support.

\section{Author details}

${ }^{1}$ Centro de Biotecnologia, Universidade Federal do Rio Grande do Sul, Av. Bento Gonçalves 9500, P.O.Box 15005, Porto Alegre, 91501-970, Brazil. ${ }^{2}$ Departamento de Botânica, Instituto de Biociências, Universidade Federal do Rio Grande do Sul, Av. Bento Gonçalves 9500, Porto Alegre, 91501-970, Brazil.

\section{Authors' contributions}

FKR designed the experiments and drafted the manuscript. FKR performed the alignments, phylogenetic, motif composition, promoter and microarray expression analyses, and participated in qPCR experiments. RAS, PKM, ERS and KLL performed qPCR experiments. FKR and RAS analyzed qPCR data. RAS helped drafting the manuscript. JPF conceived and coordinated the study and prepared the final manuscript. All authors read and approved the final manuscript.

\section{Received: 4 February 2010 Accepted: 25 January 2011}

Published: 25 January 2011

\section{References}

1. Bowers JE, Chapman BA, Rong J, Paterson AH: Unravelling angiosperm genome evolution by phylogenetic analysis of chromosomal duplication events. Nature 2003, 422:433-438.

2. Jaillon O, Aury JM, Noel B, Policriti A, Clepet C, Casagrande A, Choisne N, Aubourg S, Vitulo N, Jubin C, Vezzi A, Legeai F, Hugueney P, Dasilva C, Horner D, Mica $E$, Jublot $D$, Poulain J, Bruyère $C$, Billault $A$, Segurens $B$, Gouyvenoux M, Ugarte E, Cattonaro F, Anthouard V, Vico V, Del Fabbro C, Alaux M, Di Gaspero G, Dumas V, Felice N, Paillard S, Juman I, Moroldo M, Scalabrin S, Canaguier A, Le Clainche I, Malacrida G, Durand E, Pesole G, Laucou V, Chatelet P, Merdinoglu D, Delledonne M, Pezzotti M, Lecharny A, Scarpelli C, Artiguenave F, Pè ME, Valle G, Morgante M, Caboche M, AdamBlondon AF, Weissenbach J, Quétier F, Wincker P, French-Italian Public Consortium for Grapevine Genome Characterization: The grapevine genome sequence suggests ancestral hexaploidization in major angiosperm phyla. Nature 2007, 449:463-467.

3. Paterson AH, Bowers JE, Chapman BA: Ancient polyploidization predating divergence of the cereals, and its consequences for comparative genomics. Proc Natl Acad Sci USA 2004, 101:9903-9908.

4. Tang H, Bowers JE, Wang X, Paterson AH: Angiosperm genome comparisons reveal early polyploidy in the monocot lineage. Proc Natl Acad Sci USA 2010, 107:472-477.

5. Otto SP, Whitton J: Polyploid incidence and evolution. Annu Rev Genet 2010, 34:401-437.

6. Ohno S: Evolution by gene duplication New York: Springer; 1970.

7. Force A, Lynch M, Pickett FB, Amores A, Yan Y, Postlethwait J: Preservation of duplicate genes by complementary, degenerative mutations. Genetics 1999, 151:1531-1545.

8. Chapman BA, Bowers JE, Feltus FA, Paterson AH: Buffering of crucial functions by paleologous duplicated genes may contribute cyclicality to angiosperm genome duplication. Proc Natl Acad Sci USA 2006, 103:2730-2735.

9. Wang $\mathrm{X}$, Tang $\mathrm{H}$, Bowers JE, Feltus FA, Paterson AH: Extensive concerted evolution of rice paralogs and the road to regaining independence. Genetics 2007, 177:1753-1763.

10. Lockton S, Gaut BS: Plant conserved non-coding sequences and paralogue evolution. Trends Genet 2005, 21:60-65.

11. Wang X, Shi X, Hao B, Ge S, Luo J: Duplication and DNA segmental loss in the rice genome: implications for diploidization. New Phytol 2005, 165:937-946.

12. Gu Z, Nicolae D, Lu HH, Li WH: Rapid divergence in expression between duplicate genes inferred from microarray data. Trends Genet 2002, 18:609-613.

13. Xu S, Clark T, Zheng H, Vang S, Li R, Wong GK, Wang J, Zheng X: Gene conversion in the rice genome. BMC Genomics 2008, 9:93.

14. Paterson AH, Bowers JE, Bruggmann R, Dubchak I, Grimwood J, Gundlach H, Haberer G, Hellsten U, Mitros T, Poliakov A, Schmutz J,
Spannagl M, Tang H, Wang X, Wicker T, Bharti AK, Chapman J, Feltus FA, Gowik U, Grigoriev IV, Lyons E, Maher CA, Martis M, Narechania A, Otillar RP, Penning BW, Salamov AA, Wang Y, Zhang L, Carpita NC, Freeling M, Gingle AR, Hash CT, Keller B, Klein P, Kresovich S, McCann MC, Ming R, Peterson DG, Mehboob-ur-Rahman, Ware D, Westhoff P, Mayer KF, Messing J, Rokhsar DS: The Sorghum bicolor genome and the diversification of grasses. Nature 2009, 457:551-556.

15. Wang $X$, Tang H, Bowers JE, Paterson AH: Comparative inference of illegitimate recombination between rice and sorghum duplicated genes produced by polyploidization. Genome Res 2009, 19:1026-1032.

16. Yu J, Wang J, Lin W, Li S, Li H, Zhou J, Ni P, Dong W, Hu S, Zeng C, Zhang J, Zhang Y, Li R, Xu Z, Li S, Li X, Zheng H, Cong L, Lin L, Yin J, Geng J, Li G, Shi J, Liu J, Lv H, Li J, Wang J, Deng Y, Ran L, Shi X, Wang X, Wu Q, Li C, Ren X, Wang J, Wang X, Li D, Liu D, Zhang X, Ji Z, Zhao W, Sun $Y$, Zhang Z, Bao J, Han Y, Dong L, Ji J, Chen P, Wu S, Liu J, Xiao Y, Bu D, Tan J, Yang L, Ye C, Zhang J, Xu J, Zhou Y, Yu Y, Zhang B, Zhuang S, Wei H, Liu B, Lei M, Yu H, Li Y, Xu H, Wei S, He X, Fang L, Zhang Z, Zhang Y, Huang $X$, Su Z, Tong W, Li J, Tong Z, Li S, Ye J, Wang L, Fang L, Lei T, Chen C, Chen H, Xu Z, Li H, Huang H, Zhang F, Xu H, Li N, Zhao C, Li S, Dong L, Huang Y, Li L, Xi Y, Qi Q, Li W, Zhang B, Hu W, Zhang Y, Tian X, Jiao Y, Liang X, Jin J, Gao L, Zheng W, Hao B, Liu S, Wang W, Yuan L, Cao M, McDermott J, Samudrala R, Wang J, Wong GK, Yang H: The Genomes of Oryza sativa: a history of duplications. PLoS Biol 2009, 3:e38.

17. Salse J, Bolot S, Throude M, Jouffe V, Piegu B, Quraishi UM, Calcagno T, Cooke R, Delseny M, Feuillet C: Identification and characterization of shared duplications between rice and wheat provide new insight into grass genome evolution. Plant Cell 2008, 20:11-24.

18. Rice Chromosomes 11 and 12 Sequencing Consortia: The sequence of rice chromosomes 11 and 12, rich in disease resistance genes and recent gene duplications. BMC Biol 2005, 3:20.

19. Jacquemin J, Laudie M, Cooke R: A recent duplication revisited: phylogenetic analysis reveals an ancestral duplication highly-conserved throughout the Oryza genus and beyond. BMC Plant Biology 2009, 9:146.

20. Yu J, Hu S, Wang J, Wong GK, Li S, Liu B, Deng Y, Dai L, Zhou Y, Zhang X, Cao M, Liu J, Sun J, Tang J, Chen Y, Huang X, Lin W, Ye C, Tong W, Cong L, Geng J, Han Y, Li L, Li W, Hu G, Huang X, Li W, Li J, Liu Z, Li L, Liu J, Qi Q, Liu J, Li L, Li T, Wang X, Lu H, Wu T, Zhu M, Ni P, Han H, Dong W, Ren X, Feng X, Cui P, Li X, Wang H, Xu X, Zhai W, Xu Z, Zhang J, He S, Zhang J, Xu J, Zhang K, Zheng X, Dong J, Zeng W, Tao L, Ye J, Tan J, Ren X, Chen X, He J, Liu D, Tian W, Tian C, Xia H, Bao Q, Li G, Gao H, Cao T, Wang J, Zhao W, Li P, Chen W, Wang X, Zhang Y, Hu J, Wang J, Liu S, Yang J, Zhang G, Xiong Y, Li Z, Mao L, Zhou C, Zhu Z, Chen R, Hao B, Zheng W, Chen S, Guo W, Li G, Liu S, Tao M, Wang J, Zhu L, Yuan L, Yang H: A draft sequence of the rice genome (Oryza sativa L. ssp. indica). Science 2002, 296:79-92.

21. Goff SA, Ricke D, Lan TH, Presting G, Wang R, Dunn M, Glazebrook J, Sessions A, Oeller P, Varma H, Hadley D, Hutchison D, Martin C, Katagiri F, Lange BM, Moughamer T, Xia Y, Budworth P, Zhong J, Miguel T, Paszkowski U, Zhang S, Colbert M, Sun WL, Chen L, Cooper B, Park S, Wood TC, Mao L, Quail P, Wing R, Dean R, Yu Y, Zharkikh A, Shen R, Sahasrabudhe S, Thomas A, Cannings R, Gutin A, Pruss D, Reid J, Tavtigian S, Mitchell J, Eldredge G, Scholl T, Miller RM, Bhatnagar S, Adey N, Rubano T, Tusneem N, Robinson R, Feldhaus J, Macalma T, Oliphant A, Briggs S: A draft sequence of the rice genome (Oryza sativa L. ssp. japonica). Science 2002, 296:92-100.

22. Yuan Q, Ouyang S, Liu J, Suh B, Cheung F, Sultana R, Lee D, Quackenbush J, Buell CR: The TIGR rice genome annotation resource: annotating the rice genome and creating resources for plant biologists. Nucleic Acids Res 2003, 31:229-233.

23. Ohyanagi $H$, Tanaka T, Sakai H, Shigemoto $Y$, Yamaguchi K, Habara T, Fujii $Y$, Antonio BA, Nagamura Y, Imanishi T, Ikeo K, Itoh T, Gojobori T, Sasaki T: The Rice Annotation Project Database (RAP-DB): hub for Oryza sativa ssp. japonica genome information. Nucleic Acids Res 2006, , 34 Database: D741-4.

24. An $G$, Jeong $D H$, Jung $K H$, Lee $S$ : Reverse genetic approaches for functional genomics of rice. Plant Mol Biol 2005, 59:111-23.

25. White PJ, Broadley MR: Biofortification of crops with seven mineral elements often lacking in human diets-iron, zinc, copper, calcium, magnesium, selenium and iodine. New Phytol 2009, 182:49-84.

26. Zhao FJ, McGrath SP: Biofortification and phytoremediation. Curr Opin Plant Biol 2009, 12:373-80. 
27. Walker EL, Connolly EL: Time to pump iron: iron-deficiency-signaling mechanisms of higher plants. Curr Opin Plant Biol 2008, 11:530-5.

28. Hänsch $R$, Mendel RR: Physiological functions of mineral micronutrients (Cu, Zn, Mn, Fe, Ni, Mo, B, Cl). Curr Opin Plant Biol 2009, 12:259-66.

29. Palmer $\mathrm{CM}$, Guerinot ML: Facing the challenges of $\mathrm{Cu}, \mathrm{Fe}$ and $\mathrm{Zn}$ homeostasis in plants. Nat Chem Biol 2009, 5:333-340.

30. Kobayashi T, Itai RN, Ogo Y, Kakei Y, Nakanishi H, Takahashi M, Nishizawa NK: The rice transcription factor IDEF1 is essential for the early response to iron deficiency, and induces vegetative expression of late embryogenesis abundant genes. Plant J 2009, 60:948-961.

31. Sperotto RA, Ricachenevsky FK, Duarte GL, Boff T, Lopes KL, Sperb ER, Grusak MA, Fett JP: Identification of up-regulated genes in flag leaves during rice grain filling and characterization of OSNAC5, a new ABAdependent transcription factor. Planta 2009, 230:985-1002.

32. Wirth J, Poletti S, Aeschlimann B, Yakandawala N, Drosse B, Osorio S, Tohge T, Fernie AR, Günther D, Gruissem W, Sautter C: Rice endosperm iron biofortification by targeted and synergistic action of nicotianamine synthase and ferritin. Plant Biotechnol J 2009, 7:631-44.

33. Marschner H: Mineral nutrition of higher plants London: Academic Press; 1995

34. Haydon MJ, Cobbett CS: A novel major facilitator superfamily protein at the tonoplast influences zinc tolerance and accumulation in Arabidopsis Plant Physiol 2007, 143:1705-1719.

35. Kawachi M, Kobae $Y$, Mori H, Tomioka R, Lee $Y$, Maeshima M: A mutant strain Arabidopsis thaliana that lacks vacuolar membrane zinc transporter MTP1 revealed latent tolerance to excessive zinc. Plant Cell Physiol 2009, 50:1156-1170.

36. Stein RJ, Duarte GL, Spohr MG, Lopes SIG, Fett JP: Distinct physiological responses of two rice cultivars subjected to iron toxicity under field conditions. Ann Appl Biol 2009, 154:269-277

37. Stein RJ, Ricachenevsky FK, Fett JP: Differential regulation of the two rice ferritin genes (OsFER1 and OsFER2). Plant Sci 2009, 177:563-569.

38. Eide D, Broderius M, Fett J, Guerinot ML: A novel iron-regulated metal transporter from plants identified by functional expression in yeast. Proc Natl Acad Sci USA 1996, 93:5624-5628.

39. Lee $\mathrm{S}, \mathrm{An} \mathrm{G}$ : Over-expression of OsIRT1 leads to increased iron and zinc accumulations in rice. Plant Cell Environ 2009, 32:408-416.

40. Aoyama T, Kobayashi T, Takahashi M, Nagasaka S, Usuda K, Kakei Y, Ishimaru Y, Nakanishi H, Mori S, Nishizawa NK: OsYSL18 is a rice iron(III)deoxymugineic acid transporter specifically expressed in reproductive organs and phloem of lamina joints. Plant Mol Biol 2009, 70:681-92.

41. Lee S, Chiecko JC, Kim SA, Walker EL, Lee Y, Guerinot ML, An G: Disruption of OsYSL15 leads to iron inefficiency in rice plants. Plant Physiol 2009, 150:786-800.

42. Morrissey J, Baxter IR, Lee J, Li L, Lahner B, Grotz N, Kaplan J, Salt DE, Guerinot ML: The ferroportin metal efflux proteins function in iron and cobalt homeostasis in Arabidopsis. Plant Cell 2009, 21:3326-3338.

43. Morel M, Crouzet J, Gravot A, Auroy P, Leonhardt N, Vavasseur A, Richaud P. AtHMA3, a P1B-ATPase allowing $\mathrm{Cd} / \mathrm{Zn} / \mathrm{Co} / \mathrm{Pb}$ vacuolar storage in Arabidopsis. Plant Physiol 2009, 149:894-904

44. Simmons CR, Fridlender M, Navarro PA, Yalpani N: A maize defenseinducible gene is a major facilitator superfamily member related to bacterial multidrug resistance efflux antiporters. Plant Mol Biol 2003, 352:433-446.

45. Saier MH Jr, Beatty JT, Goffeau A, Harley KT, Heijne WH, Huang SC, Jack DL, Jähn PS, Lew K, Liu J, Pao SS, Paulsen IT, Tseng TT, Virk PS: The major facilitator superfamily. J Mol Microbiol Biotechnol 1999, 1:257-279.

46. Waters BM, Grusak MA: Quantitative trait locus mapping for seed mineral concentrations in two Arabidopsis thaliana recombinant inbred populations. New Phytol 2008, 179:1033-1047.

47. Tauris B, Borg S, Gregersen PL, Holm PB: A roadmap for zinc trafficking in the developing barley grain based on laser capture microdissection and gene expression profiling. J Exp Bot 2009, 60:1333-1347.

48. Gross J, Stein RJ, Fett-Neto AG, Fett JP: Iron homeostasis related genes in rice. Genet Mol Biol 2003, 26:477-497.

49. Bailey TL, Williams N, Misleh C, Li WW: MEME: discovering and analyzing DNA and protein sequence motifs. Nucleic Acids Res 2006, 34:W369-373.

50. López-Millán AF, Ellis DR, Grusak MA: Identification and characterization of several new members of the ZIP family of metal ion transporters in Medicago truncatula. Plant Mol Biol 2004, 54:583-96.
51. Yang $X$, Huang J, Jiang $Y$, Zhang HS: Cloning and functional identification of two members of the ZIP (Zrt, Irt-like protein) gene family in rice (Oryza sativa L.). Mol Biol Rep 2009, 36:281-287.

52. Montanini B, Blaudez D, Jeandroz S, Sanders D, Chalot M: Phylogenetic and functional analysis of the Cation Diffusion Facilitator (CDF) family: improved signature and prediction of substrate specificity. BMC Genomics 2007, 8:107.

53. Kankainen $\mathrm{M}, \mathrm{Holm} \mathrm{L}$ : POCO: discovery of regulatory patterns from promoters of oppositely expressed gene sets. Nucleic Acids Res 2005, 33: W427-31.

54. Kobayashi T, Ogo Y, Itai RN, Nakanishi H, Takahashi M, Mori S, Nishizawa NK The transcription factor IDEF1 regulates the response to and tolerance of iron deficiency in plants. Proc Natl Acad Sci USA 2007, 104:19150-19155.

55. Morris RT, O'Connor TR, Wyrick JJ: Osiris: an integrated promoter database for Oryza sativa L. Bioinformatics 2008, 24:2915-2917.

56. Kobayashi T, Suzuki M, Inoue H, Itai RN, Takahashi M, Nakanishi H, Mori S, Nishizawa NK: Expression of iron-acquisition-related genes in irondeficient rice is co-ordinately induced by partially conserved irondeficiency-responsive elements. J Exp Bot 2005, 56:1305-1316.

57. Long TA, Tsukagoshi H, Busch W, Lahner B, Salt DE, Benfey PN: The bHLH Transcription Factor POPEYE Regulates Response to Iron Deficiency in Arabidopsis Roots. Plant Cell 2010, 22:2219-36.

58. Buckhout TJ, Yang TJ, Schmidt W: Early iron-deficiency-induced transcriptional changes in Arabidopsis roots as revealed by microarray analyses. BMC Genomics 2009, 10:147

59. Ishimaru Y, Suzuki M, Ogo Y, Takahashi M, Nakanishi H, Mori S, Nishizawa NK: Synthesis of nicotianamine and deoxymugineic acid is regulated by OsIRO2 in Zn excess rice plants. Soil Sci Plant Nut 2008, 54:417-423.

60. Bughio N, Yamaguchi H, Nishizawa NK, Nakanishi H, Mori S: Cloning an iron-regulated metal transporter from rice. J Exp Bot 2002, 53:1677-1682.

61. Ishimaru Y, Suzuki M, Tsukamoto T, Suzuki K, Nakazono M, Kobayashi T, Wada Y, Watanabe S, Matsuhashi S, Takahashi M, Nakanishi H, Mori S, Nishizawa NK: Rice plants take up iron as an $\mathrm{Fe}^{3+}$-phytosiderophore and as $\mathrm{Fe}^{2+}$. Plant J 2006, 45:335-346.

62. Ogo $Y$, Itai RN, Nakanishi H, Inoue $H$, Kobayashi T, Suzuki M, Takahashi M, Mori S, Nishizawa NK: Isolation and characterization of IRO2, a novel ironregulated bHLH transcription factor in graminaceous plants. J Exp Bot 2006, 57:2867-2878.

63. Zimmermann P, Hennig L, Gruissem W: Gene-expression analysis and network discovery using Genevestigator. Trends Plant Sci 2005, 10:407-409.

64. Schmittgen TD, Livak KJ: Analyzing real-time PCR data by the comparative $C_{t}$ method. Nat Protoc 2008, 3:1101-1108.

65. Norton GJ, Lou-Hing DE, Meharg AA, Price AH: Rice-arsenate interactions in hydroponics: whole genome transcriptional analysis. J Exp Bot 2008, 59:2267-2276.

66. Cui L, Wall PK, Leebens-Mack JH, Lindsay BG, Soltis DE, Doyle JJ, Soltis PS, Carlson JE, Arumuganathan K, Barakat A, Albert VA, Ma H, dePamphilis CW: Widespread genome duplications throughout the history of flowering plants. Genome Res 2007, 16:738-749.

67. Flagel LE, Wendel JF: Gene duplication and evolutionary novelty in plants. New Phytol 2009, 183:557-564.

68. Moore RC, Purugganan MD: The evolutionary dynamics of plant duplicate genes. Curr Opin Plant Biol 2005, 8:122-128.

69. Duarte JM, Cui L, Wall PK, Zhang Q, Zhang X, Leebens-Mack J, Ma H, Altman N, dePamphilis CW: Expression pattern shifts following duplication indicative of subfunctionalization and neofunctionalization in regulatory genes of Arabidopsis. Mol Biol Evol 2006, 23:469-478.

70. Ha M, Kim ED, Chen ZJ: Duplicate genes increase expression diversity in closely related species and allopolyploids. Proc Natl Acad Sci USA 2009, 106:2295-2300.

71. Hoffman AA, Rieseberg LH: Revisiting the impact of inversions in evolution: frompopulation geneticmarkers to drivers of adaptive shifts and speciation? Ann Rev Ecol Evol Syst 2008, 39:21-42.

72. Feder $J$, Nosil P: Chromosomal inversions and species differences: when are genes affecting adaptive divergence and reproductive isolation expected to reside within inversions? Evolution 2009, 63:3061-3075.

73. Throude M, Bolot S, Bosio M, Pont C, Sarda X, Quraishi UM, Bourgis F, Lessard P, Rogowsky P, Ghesquiere A, Murigneux A, Charmet G, Perez P, 
Salse J: Structure and expression analysis of rice paleo duplications. Nucleic Acids Res 2009, 37:1248-1259.

74. Yim WC, Lee BM, Jang CS: Expression diversity and evolutionary dynamics of rice duplicate genes. Mol Genet Genomics 2009, 281:483-493.

75. Blanc $\mathrm{G}$, Wolfe $\mathrm{KH}$ : Functional divergence of duplicated genes formed by polyploidy during Arabidopsis evolution. Plant Cell 2004, 16:1679-1691.

76. Rizzon C, Ponger L, Gaut BS: Striking similarities in the genomic distribution of tandemly arrayed genes in Arabidopsis and rice. PLOS Comput Biol 2006, 2:e115.

77. Schmid M, Davison TS, Henz SR, Pape UJ, Demar M, Vingron M, Schölkopf B, Weigel D, Lohmann JU: A gene expression map of Arabidopsis thaliana development. Nat Genet 2005, 37:501-506.

78. Ishimaru Y, Bashir K, Fujimoto M, An G, Itai RN, Tsutsumi N, Nakanishi H, Nishizawa NK: Rice-specific mitochondrial iron-regulated gene (MIR) plays an important role in iron homeostasis. Mol Plant 2009, 2:1059-1066.

79. Cassin G, Mari S, Curie C, Briat JF, Czernic P: Increased sensitivity to iron deficiency in Arabidopsis thaliana overaccumulating nicotianamine. J Exp Bot 2009, 60:1249-1259.

80. Ogo Y, Itai RN, Nakanishi H, Kobayashi T, Takahashi M, Mori S, Nishizawa NK: The rice bHLH protein OsIRO2 is an essential regulator of the genes involved in Fe uptake under Fe-deficient conditions. Plant J 2007, 51:366-377.

81. Counce PA, Keisling TC, Mitchell AJ: A uniform, objective and adaptative system for expressing rice development. Crop Sci 2000, 40:436-443.

82. Eddy SR: Profile hidden Markov models. Bioinformatics 1998, 14:755-63.

83. Schuster-Böckler B, Schultz J, Rahmann S: HMM Logos for visualization of protein families. BMC Bioinformatics 2004, 5:7.

84. Schuster-Böckler B, Bateman A: Visualizing profile-profile alignment: pairwise HMM logos. Bioinformatics 2005, 21:2912-3.

85. Tamura K, Dudley J, Nei M, Kumar S: MEGA4: Molecular Evolutionary Genetics Analysis (MEGA) software version 4.0. Mol Biol Evol 2007, 24:1596-1599.

86. Huelsenbeck JP, Ronquist F: MRBAYES. Bayesian inference of phylogeny. Bioinformatics 2001, 17:754-755.

87. Ronquist F, Huelsenbeck JP: MrBayes 3: Bayesian phylogenetic inference under mixed models. Bioinformatics 2003, 19:1572-1574.

88. Nix DA, Eisen MB: GATA: a graphic alignment tool for comparative sequence analysis. BMC Bioinformatics 2005, 6:9.

89. Livak KJ, Schmittgen TD: Analysis of relative gene expression data using real-time quantitative PCR and the $2^{-\Delta \Delta C t}$ method. Methods 2001, 25:402-408.

90. Ramakers C, Ruijter JM, Deprez RH, Moorman AF: Assumption-free analysis of quantitative real-time polymerase chain reaction (PCR) data. Neurosci Lett 2003, 339:62-6.

91. Jain M, Nijhawan A, Tyagi AK, Khurana JP: Validation of housekeeping genes as internal control for studying gene expression in rice by quantitative real-time PCR. Biochem Biophys Res Commun 2006, 345:646-651.

doi:10.1186/1471-2229-11-20

Cite this article as: Ricachenevsky et al: ZINC-INDUCED FACILITATOR-

LIKE family in plants: lineage-specific expansion in monocotyledons and conserved genomic and expression features among rice (Oryza sativa) paralogs. BMC Plant Biology 2011 11:20.

\section{Submit your next manuscript to BioMed Central and take full advantage of:}

- Convenient online submission

- Thorough peer review

- No space constraints or color figure charges

- Immediate publication on acceptance

- Inclusion in PubMed, CAS, Scopus and Google Scholar

- Research which is freely available for redistribution

Submit your manuscript at www.biomedcentral.com/submit
Ciomed Central 